\title{
Chemical Compositions, Mosquito Larvicidal and Antimicrobial Activities of Leaf Essential Oils of Eleven Species of Lauraceae from Vietnam
}

\author{
Dao Thi Minh Chau ${ }^{1}$, Nguyen Thanh Chung ${ }^{2}$, Le Thi Huong ${ }^{3}{ }^{(0)}$, Nguyen Huy Hung ${ }^{4}{ }^{(}$, \\ Isiaka A. Ogunwande ${ }^{5}$ (D), Do Ngoc Dai ${ }^{2,6, *(D)}$ and William N. Setzer ${ }^{7,8, *(D)}$ \\ 1 Institute of Biochemical Technology and Environment, Vinh University, 182 Le Duan, \\ Vinh City 4300, Nghệ An Province, Vietnam; daochau27@gmail.com \\ 2 Graduate University of Science and Technology, Vietnam Academy of Science and Technology, \\ 18-Hoang Quoc Viet, Cau Giay 10072, Hanoi, Vietnam; chungphoat@gmail.com \\ 3 School of Natural Science Education, Vinh University, 182 Le Duan, \\ Vinh City 4300, Nghệ An Province, Vietnam; lehuong223@gmail.com \\ 4 Center for Advanced Chemistry, Institute of Research and Development, Duy Tan University, \\ 03 Quang Trung, Da Nang 5000, Vietnam; nguyenhuyhung@duytan.edu.vn \\ 5 Foresight Institute of Research and Translation, University Road, Aleku Area, Osogbo 230271, Nigeria; \\ isiakaogunwande@gmail.com \\ 6 Faculty of Agriculture, Forestry and Fishery, Nghe An College of Economics, 51-Ly Tu Trong, \\ Vinh City 4300, Nghe An Province, Vietnam \\ 7 Department of Chemistry, University of Alabama in Huntsville, Huntsville, AL 35899, USA \\ 8 Aromatic Plant Research Center, 230 N 1200 E, Suite 100, Lehi, UT 84043, USA \\ * Correspondence: daidn23@gmail.com (D.N.D.); wsetzer@chemistry.uah.edu (W.N.S.)
}

Received: 23 April 2020; Accepted: 7 May 2020; Published: 10 May 2020

\begin{abstract}
The Lauraceae is a family rich in aromatic and medicinal plants. Likewise, essential oils derived from members of this family have demonstrated a myriad of biological activities. It is hypothesized that members of the Lauraceae from Vietnam will yield essential oils that may be useful in controlling mosquito populations and treating microbial infections. In this work, the leaf essential oils of eleven species of Lauraceae (Beilschmiedia erythrophloia, B. robusta, B. yunnanensis, Cryptocarya concinna, C. impressa, C. infectoria, Litsea viridis, Machilus balansa, M. grandifolia, Neolitsea ellipsoidea, and Phoebe angustifolia) have been obtained by hydrodistillation and the chemical compositions analyzed by gas chromatography - mass spectrometry (GC-MS) and gas chromatography with flame ionization detection (GC-FID). The essential oils were screened for larvicidal activity against Aedes aegypti, Ae. albopictus, and Culex quinquefasciatus, and for antimicrobial activity against Enterococcus faecalis, Staphylococcus aureus, Bacillus cereus, Escherichia coli, Pseudomonas aeruginosa, Salmonella enterica, and Candida albicans. The leaf essential oil of N. ellipsoidea, rich in (E)- $\beta$-ocimene (87.6\%), showed excellent larvicidal activity against $A e$. aegypti with a $24 \mathrm{~h} \mathrm{LC}_{50}$ of $6.59 \mu \mathrm{g} / \mathrm{mL}$. The leaf essential oil of C. infectoria, dominated by germacrene D (55.5\%) and bicyclogermacrene (11.4\%), exhibited remarkable larvicidal activity against $C x$. quinquefasciatus $(48 \mathrm{~h}$ $\mathrm{LC}_{50}=0.40 \mu \mathrm{g} / \mathrm{mL}$ ). N. ellipsoidea leaf essential oil also demonstrated notable antibacterial activity against $E$. faecalis and B. cereus with minimum inhibitory concentration (MIC) values of $16 \mu \mathrm{g} / \mathrm{mL}$, while the leaf essential oil of $C$. impressa showed excellent anticandidal with an MIC of $16 \mu \mathrm{g} / \mathrm{mL}$. Leaf essential oils from the Lauraceae should be considered for utilization as alternative agents for controlling mosquito populations and as antimicrobial agents.
\end{abstract}

Keywords: Beilschmiedia; Cryptocarya; Litsea; Machilus; Neolitsea; Phoebe; Aedes; Culex; antibacterial; antifungal 


\section{Introduction}

The Lauraceae is made up of around 55 genera and 3000 species of tropical and warm temperate trees and shrubs, with Southeast Asia and Brazil serving as species-rich hot spots [1]. Several members of the family are commercially important, including the avocado (Persea americana Mill.) for its fruit, bay leaf (Laurus nobilis L.) used in cooking, and the spice cinnamon (Cinnamomum verum J. Presl) [2]. Several Lauraceae species have been used medicinally, including sassafras (Sassafras albidum (Nutt.) Nees) [3] and spicebush (Lindera benzoin (L.) Blume) [4]. Many species of Lauraceae contain essential oils that have found use in the flavor and fragrance industry [5], e.g., Brazilian rosewood (Aniba rosaeodora Ducke) [6], camphor tree, ravintsara, ho leaf (Cinnamomum camphora (L.) J. Presl.) [7], and aromatic litsea (Litsea cubeba (Lour.) Pers.) [8].

Based on the utility and properties of Lauraceae essential oils, it is hypothesized that members of the Lauraceae found in Vietnam have biologically active essential oils that may be useful in controlling mosquito populations or as antimicrobial agents. Eleven species of Lauraceae from north-central Vietnam have been collected, the essential oils obtained by hydrodistillation, chemical compositions analyzed, and the oils screened for mosquito larvicidal activity and for antimicrobial activity.

The genus Beilschmiedia Nees is comprised of around 250 species of trees and shrubs [9] and are widespread in tropical Africa, Madagascar, Asia, Southeast Asia, Melanesia, Australia, New Zealand, North America, Central America, South America, and the Caribbean [10]. The phytochemistry and bioactivity of Beilschmiedia has been reviewed [11].

Beilschmiedia erythrophloia Hayata is a tree found in Taiwan, southern China, Hainan Island, and Ryukyu Islands (Japan) [12,13]. In Vietnam, the tree is found in Nghệ An, Hà Tĩnh, and Đồng Nai provinces [14]. Previous phytochemical studies of B. erythrophloia have revealed endiandric acid derivatives from the roots [15,16], the cytotoxic alkaloid beischamide from the stems [13], and a leaf essential oil rich in (E)-caryophyllene and $\alpha$-humulene [17].

Beilschmiedia robusta C.K. Allen is a tree, $10-15 \mathrm{~m}$ tall that is recorded from Guangzi, southwestern Guizhou, Xizang, and Yunnan provinces in China [12,18]. In Vietnam, the tree is found in Lào Cai, Ninh Bình, and Nghệ An provinces [14]. A perusal of the literature has revealed that there have been no previous phytochemical investigations of $B$. robusta.

Beilschmiedia yunnanensis H.H. Hu is a tree, up to $18 \mathrm{~m}$ tall and is found in Guangdong, southern Guangxi, and southern Yunnan provinces in China [12]. In Vietnam, the tree is found in Lào Cai, Nghệ An, and Hà Tĩnh provinces [14]. A literature search has revealed that there have been no previous phytochemical investigations of B. yunnanensis.

Cryptocarya R. Br. is a pantropical genus of around 300 species [19]. Cryptocarya concinna Hance (syn. Cryptocarya konishii Hayata, Cryptocarya lenticellata Lecomte, Cryptocarya microcarpa F.N. Wei) is a tree up to $18 \mathrm{~m}$ tall, and ranges from southern China (Guangdong, Guangxi, southeastern Guizhou, Hainan, Jiangxi, and Taiwan) to northern Vietnam [9,12]. In Vietnam, it has been recorded in Hà Giang, Tuyên Quang, Cao Bằng, Vĩnh Phúc, Hải Phòng, Thanh Hóa, Nghệ An, Hà Tĩnh, Thừa Thiên Huế provinces [14]. Previous investigations of the phytochemistry of $C$. concinna have shown the roots to contain cytotoxic cryptocaryone [20], the leaves to contain cytotoxic cryptoconcatones $\mathrm{K}$ and L [21], and the wood to contain cytotoxic cryptocaryone and kurzichalcolactone A and antifungal cryptocaryanone A and kurzichalcolactone B [22]. There have been no previous reports on essential oils from C. concinna.

Cryptocarya impressa Miq. is native to Vietnam, Laos, the Malay Peninsula, Borneo and Sumatra [23]. In Vietnam, the plant has been recorded in Hòa Bình, Hà Nội, Hải Dương, Ninh Bình, Nghệ An, and Gia Lai provinces [14]. To our knowledge, there have been no reports on the phytochemistry of C. impressa.

Cryptocarya infectoria (Blume) Miq. (syn. Cylicodaphne infectoria Blume) is a tree up to $33 \mathrm{~m}$ tall that is native to Indo-China and Malesia [24-26]. In Vietnam, this tree is found in Lào Cai, Phú Thọ, Vĩnh Phúc, Thanh Hoá, Nghệ An, Hà Tĩnh, and Thừa Thiên Huế provinces [14]. The cytotoxic dihydrochalcones, cryptocaryone and infectocaryone, and the flavonoids cryptocaryanones A and B 
have been isolated from the methanol bark extract of $C$. infectoria $[27,28]$. The isoquinoline alkaloids atherosperminine, $N$-methylisococlaurine, and $N$-methyllaurotetanine have also been isolated from the bark of C. infectoria [29]. There have been apparently no essential oil analyses on this plant, however.

The genus Litsea Lam. consists of around 300 species distributed in tropical and warm subtropical regions of Asia, Australia, and the Americas [19]. Litsea viridis H. Liu is a small tree, 3-6 m tall, found in south-eastern Yunnan province (China) and Cao Bằng, Nghệ An, Đà Nẵng, and Đắk Lắk provinces (Vietnam) [12,14]. There do not seem to be any previous studies on the phytochemistry of this plant.

The genus Machilus Rumph. ex Nees is comprised of around 100 species distributed in southern and south-eastern Asia [12,14]. Machilus balansae (Airy Shaw) F.N. Wei \& S.C. Tang (syn. Persea balansae Airy Shaw) is endemic to Vietnam and is generally found at low elevations in north Vietnam [30]. Machilus grandifolia S.K. Lee \& F.N. Wei is now regarded as a new synonym of M. balansae [30]. To our knowledge, there have been no phytochemical studies reported on M. balansae or M. grandifolia.

The genus Neolitsea (Benth.) Merr. Contains around 85 species distributed from Indo-Malaysia to East Asia [12,14]. Neolitsea ellipsoidea K.C. Allen is a tree up to $30 \mathrm{~m}$ in height [31]. The species has been recorded in Hainan (China) and Vietnam (Hoà Bình, Quảng Ninh, Hà Tĩnh, and Gia Lai provinces). To our knowledge there have been no reports on the phytochemistry of this species.

There are around 100 species in the genus Phoebe Nees [19], which range from the Neotropics (Mexico, south to Brazil, Bolivia, and Argentina) and Southeast Asia (southern China, Vietnam, Thailand, Myanmar, Cambodia, and Singapore), as well as Indonesia, New Guinea, and India [9].

Phoebe angustifolia Meisn. is a small shrub found in southeastern Yunnan (China), Myanmar, India, and Vietnam [12]. In Vietnam, the species has been recorded in Thanh Hóa, Nghệ An, Thừa Thiên Huế, and Quảng Nam provinces [14]. The leaf essential oil of P. angustifolia from Vietnam has been reported, which showed the major components to be spathulenol $(17.0 \%)$, palmitic acid $(13.0 \%)$, sabinene $(6.4 \%)$, bicyclogermacrene (5.9\%), and artemisia triene (5.1\%) [32].

\section{Results and Discussion}

The essential oil collection details and yields are summarized in Table 1.

Table 1. Plant collection and hydrodistillation details of Lauraceae from Vietnam.

\begin{tabular}{|c|c|c|c|c|c|}
\hline Plant Species & $\begin{array}{l}\text { Vietnamese } \\
\text { Name }\end{array}$ & Collection Site & $\begin{array}{l}\text { Voucher } \\
\text { Number }\end{array}$ & $\begin{array}{l}\text { Collection } \\
\text { Month/Year }\end{array}$ & $\begin{array}{c}\text { Yield } \\
(\%, \mathrm{v} / \mathrm{w})\end{array}$ \\
\hline $\begin{array}{l}\text { Beilschmiedia erythrophloia } \\
\text { Hayata }\end{array}$ & Chắp, Kết gỗ đỏ & $\begin{array}{c}\text { Pù Hoạt Nature Reserve; } 19^{\circ} 41^{\prime} 40^{\prime \prime} \mathrm{N}, \\
104^{\circ} 49^{\prime} 31^{\prime \prime} \text { E, elev, } 678 \mathrm{~m}\end{array}$ & 803 & $7 / 2019$ & 0.12 \\
\hline Beilschmiedia robusta C.K. Allen & $\begin{array}{l}\text { Chắp to khỏ, } \\
\text { Két to khỏe }\end{array}$ & $\begin{array}{c}\text { Pù Hoạt Nature Reserve; } 19^{\circ} 41^{\prime} 37^{\prime \prime} \mathrm{N}, \\
104^{\circ} 49^{\prime} 30^{\prime \prime} \text { E, elev. } 677 \mathrm{~m}\end{array}$ & 827 & $9 / 2019$ & 0.14 \\
\hline $\begin{array}{c}\text { Beilschmiedia yunnanensis } \\
\text { H.H. Hu }\end{array}$ & $\begin{array}{l}\text { Chắp vân nam, } \\
\text { Két vân nam, } \\
\text { Mong vân nam }\end{array}$ & $\begin{array}{c}\text { Vũ Quang National park; } 18^{\circ} 17^{\prime} 15^{\prime \prime} \mathrm{N}, \\
105^{\circ} 21^{\prime} 39^{\prime \prime} \text { E, elev. } 153 \mathrm{~m}\end{array}$ & 799 & $7 / 2019$ & 0.15 \\
\hline \multirow[t]{2}{*}{ Cryptocarya concinna Hance } & \multirow{2}{*}{$\begin{array}{l}\text { Ẩn hạch quả } \\
\text { vàng, Mò quả } \\
\text { vàng, Kháo }\end{array}$} & $\begin{array}{c}\text { Nam Đông District, Thừa Thiên Huế } \\
\text { Province; } 16^{\circ} 13^{\prime} 9^{\prime \prime} \mathrm{N}, 107^{\circ} 43^{\prime} 28^{\prime \prime} \mathrm{E}, \\
\text { elev. } 124 \mathrm{~m}\end{array}$ & 791 & $7 / 2019$ & 0.33 \\
\hline & & $\begin{array}{c}\text { Pù Hoạt Nature Reserve; } 19^{\circ} 42^{\prime} 18^{\prime \prime} \mathrm{N}, \\
104^{\circ} 49^{\prime} 42^{\prime \prime} \text { E, elev. } 648 \mathrm{~m}\end{array}$ & 801 & $7 / 2019$ & 0.36 \\
\hline $\begin{array}{l}\text { Cryptocarya impressa Miq. } \\
\text { Syn.: Cryptocarya venosa Meisn. } \\
\text { ex Hook.f. }\end{array}$ & $\begin{array}{l}\text { Mò quả to, Mò } \\
\text { quả xanh, Ẩn } \\
\text { hạch quả to }\end{array}$ & $\begin{array}{c}\text { Pù Hoạt Nature Reserve; } 19^{\circ} 42^{\prime} 18^{\prime \prime} \mathrm{N}, \\
104^{\circ} 49^{\prime} 42^{\prime \prime} \text { E, elev. } 648 \mathrm{~m}\end{array}$ & 826 & $9 / 2019$ & 0.22 \\
\hline $\begin{array}{l}\text { Cryptocarya infectoria } \\
\text { (Blume) Miq. } \\
\text { Syn.: Caryodaphne infectoria } \\
\text { Blume }\end{array}$ & $\begin{array}{l}\text { Cà đuối nhuộm, } \\
\text { Ẩn hạch nhuộm, } \\
\text { Cà đuối tai nghé }\end{array}$ & $\begin{array}{c}\text { Pù Hoạt Nature Reserve; } 19^{\circ} 42^{\prime} 18^{\prime \prime} \mathrm{N}, \\
104^{\circ} 49^{\prime} 42^{\prime \prime} \text { E, elev. } 648 \mathrm{~m}\end{array}$ & 767 & $4 / 2019$ & 0.25 \\
\hline Litsea viridis $\mathrm{H}$. Liou & Bời lời xanh & $\begin{array}{c}\text { Pù Hoạt Nature Reserve; } 19^{\circ} 42^{\prime} 18^{\prime \prime} \mathrm{N}, \\
104^{\circ} 49^{\prime} 42^{\prime \prime} \text { E, elev. } 648 \mathrm{~m}\end{array}$ & 806 & $8 / 2019$ & 0.21 \\
\hline
\end{tabular}


Table 1. Cont

\begin{tabular}{|c|c|c|c|c|c|}
\hline Plant Species & $\begin{array}{l}\text { Vietnamese } \\
\text { Name }\end{array}$ & Collection Site & $\begin{array}{l}\text { Voucher } \\
\text { Number }\end{array}$ & $\begin{array}{l}\text { Collection } \\
\text { Month/Year }\end{array}$ & $\begin{array}{c}\text { Yield } \\
(\%, v / w)\end{array}$ \\
\hline $\begin{array}{c}\text { Machilus balansa (Airy Shaw) } \\
\text { F.N. Wei \& S.C. Tang } \\
\text { Syn.: Persea balansae } \\
\text { Airy Shaw }\end{array}$ & $\begin{array}{l}\text { Kháo balansa, } \\
\text { Rè balansa }\end{array}$ & $\begin{array}{c}\text { Pù Mát National Park; } 18^{\circ} 58^{\prime} 14^{\prime \prime} \mathrm{N} \text {, } \\
104^{\circ} 48^{\prime} 2^{\prime \prime} \text { E, elev. } 376 \mathrm{~m}\end{array}$ & 828 & $9 / 2019$ & 0.42 \\
\hline $\begin{array}{c}\text { Machilus grandifolia S.K. Lee \& } \\
\text { F.N. Wei }\end{array}$ & Kháo lá to & $\begin{array}{c}\text { Nam Đông District, Thừa Thiên Huế } \\
\text { Province; } 16^{\circ} 13^{\prime} 9^{\prime \prime} \mathrm{N}, 107^{\circ} 43^{\prime} 28^{\prime \prime} \mathrm{E} \text {, } \\
\text { elev. } \mathrm{ZZ} \mathrm{m}\end{array}$ & 779 & $7 / 2019$ & 0.18 \\
\hline Neolitsea ellipsoidea K.C. Allen & $\begin{array}{l}\text { Nô bầu dục, Bài } \\
\text { nhài lá bầu dục, } \\
\text { Tam tầng }\end{array}$ & $\begin{array}{c}\text { Vũ Quang National park; } 18^{\circ} 17^{\prime} 15^{\prime \prime} \mathrm{N}, \\
105^{\circ} 21^{\prime} 39^{\prime \prime} \text { E, elev. } 124 \mathrm{~m}\end{array}$ & 802 & $7 / 2019$ & 0.31 \\
\hline $\begin{array}{c}\text { Phoebe angustifolia Meisn. } \\
\text { Syn.: Phoebe angustifolia var. } \\
\text { annamensis Liou }\end{array}$ & $\begin{array}{l}\text { Re trắng lá hẹp, } \\
\text { Sụ lá hẹp, Dù dà } \\
\text { mò cát }\end{array}$ & $\begin{array}{c}\text { Pù Hoạt Nature Reserve; } 19^{\circ} 49^{\prime} 7^{\prime \prime} \mathrm{N} \text {, } \\
104^{\circ} 55^{\prime} 38^{\prime \prime} \text { E, elev. } 465 \mathrm{~m}\end{array}$ & 785 & $7 / 2019$ & 0.45 \\
\hline
\end{tabular}

\subsection{Essential Oil Compositions}

The essential oil compositions of B. erythrophloia, B. robusta, and B. yunnanensis are compiled in Table 2. All three of the Beilschmiedia leaf essential oils were dominated by sesquiterpene hydrocarbons. A preponderance of sesquiterpene hydrocarbons has been previously seen in Beilschmiedia leaf essential oils from Malaysia [33] and from Costa Rica [34].

Table 2. Chemical compositions of the leaf essential oils of Beilschmiedia species collected in Vietnam.

\begin{tabular}{|c|c|c|c|c|c|}
\hline \multirow{2}{*}{$\mathrm{RI}_{\text {calc }}$} & \multirow{2}{*}{$R I_{d b}$} & \multirow{2}{*}{ Compounds } & \multicolumn{3}{|c|}{ Percent Composition } \\
\hline & & & B.e. & B.r. & B.y. \\
\hline 930 & 924 & $\alpha$-Thujene & - & 0.1 & 0.6 \\
\hline 939 & 932 & $\alpha$-Pinene & 3.2 & 2.9 & 6.0 \\
\hline 955 & 946 & Camphene & 0.2 & 0.2 & 0.2 \\
\hline 979 & 969 & Sabinene & 0.1 & 0.6 & 1.9 \\
\hline 984 & 974 & $\beta$-Pinene & 0.6 & 2.7 & 4.7 \\
\hline 992 & 988 & Myrcene & 0.5 & 0.4 & 0.8 \\
\hline 1010 & 1002 & $\alpha$-Phellandrene & 0.1 & - & 0.1 \\
\hline 1022 & 1014 & $\alpha$-Terpinene & - & 0.5 & 1.5 \\
\hline 1030 & 1020 & p-Cymene & - & 0.3 & 0.8 \\
\hline 1034 & 1024 & Limonene & 0.2 & 0.8 & 1.2 \\
\hline 1035 & 1025 & $\beta$-Phellandrene & - & 0.1 & 0.6 \\
\hline 1039 & 1032 & (Z)- $\beta$-Ocimene & 26.1 & - & 0.1 \\
\hline 1049 & 1044 & (E)- $\beta$-Ocimene & 3.6 & 0.5 & - \\
\hline 1063 & 1054 & $\gamma$-Terpinene & - & 0.9 & 2.6 \\
\hline 1094 & 1086 & Terpinolene & - & 0.4 & 0.8 \\
\hline 1117 & 1113 & (E)-4,8-Dimethylnona-1,3,7-triene & - & - & 0.2 \\
\hline 1131 & 1128 & allo-Ocimene & 0.6 & - & - \\
\hline 1188 & 1174 & Terpinen-4-ol & - & 0.5 & 1.8 \\
\hline 1200 & 1186 & $\alpha$-Terpineol & - & - & 0.2 \\
\hline 1294 & 1287 & Bornyl acetate & 0.3 & - & - \\
\hline 1348 & 1335 & $\delta$-Elemene & 1.5 & 0.3 & 0.3 \\
\hline 1360 & 1345 & $\alpha$-Cubebene & - & 0.3 & - \\
\hline 1365 & 1359 & Neryl acetate & - & - & 0.2 \\
\hline 1384 & 1373 & $\alpha$-Ylangene & - & 0.1 & - \\
\hline 1386 & 1387 & $\beta$-Cubebene & - & - & 0.1 \\
\hline 1389 & 1374 & $\alpha$-Copaene & 0.3 & 0.7 & 0.2 \\
\hline 1397 & 1390 & 7-epi-Sesquithujene & 0.5 & 0.7 & 1.0 \\
\hline 1399 & 1387 & $\beta$-Bourbonene & - & 0.9 & - \\
\hline 1404 & 1389 & $\beta$-Elemene & 1.0 & 1.0 & 0.6 \\
\hline 1425 & 1411 & cis- $\alpha$-Bergamotene & - & 0.4 & 0.4 \\
\hline
\end{tabular}


Table 2. Cont.

\begin{tabular}{|c|c|c|c|c|c|}
\hline \multirow{2}{*}{$\mathbf{R I}_{\text {calc }}$} & \multirow{2}{*}{$\mathbf{R I}_{\mathrm{db}}$} & \multirow{2}{*}{ Compounds } & \multicolumn{3}{|c|}{ Percent Composition } \\
\hline & & & B.e. & B.r. & B.y. \\
\hline 1428 & 1409 & $\alpha$-Gurjunene & - & - & 0.3 \\
\hline 1437 & 1417 & (E)-Caryophyllene & 18.3 & 22.5 & 16.2 \\
\hline 1446 & 1432 & trans- $\alpha$-bergamotene & 0.5 & 1.2 & 1.1 \\
\hline 1452 & 1437 & $\alpha$-Guaiene & - & 0.4 & 0.4 \\
\hline 1457 & 1439 & Aromadendrene & 0.7 & 1.5 & 1.8 \\
\hline 1460 & 1440 & (Z)- $\beta$-Farnesene & 0.3 & 0.2 & 0.5 \\
\hline 1466 & 1448 & cis-Muurola-3,5-diene & - & 0.2 & - \\
\hline 1471 & 1452 & $\alpha$-Humulene & 2.6 & 13.4 & 9.9 \\
\hline 1479 & 1464 & 9-epi-(E)-Caryophyllene & 0.4 & 0.5 & 21.2 \\
\hline 1488 & 1481 & $\gamma$-Curcumene & - & - & 0.2 \\
\hline 1490 & 1478 & $\gamma$-Muurolene & 0.1 & 1.9 & 0.3 \\
\hline 1494 & 1483 & $\alpha$-Amorphene & - & 0.6 & - \\
\hline 1498 & 1484 & Germacrene D & 2.7 & 20.3 & 1.1 \\
\hline 1504 & 1489 & $\beta$-Selinene & - & - & 0.2 \\
\hline 1505 & 1492 & $\delta$-Selinene & - & 0.4 & 0.2 \\
\hline 1507 & 1490 & 9-Aromadendrene & - & - & 0.9 \\
\hline 1512 & 1505 & $(E, E)-\alpha$-Farnesene & - & 1.4 & - \\
\hline 1512 & 1496 & Viridiflorene & - & 2.4 & 2.0 \\
\hline 1514 & 1500 & Bicyclogermacrene & 30.5 & 8.6 & 8.4 \\
\hline 1520 & 1514 & $\beta$-Curcumene & - & - & 0.2 \\
\hline 1521 & 1511 & $\delta$-Amorphene & 0.1 & - & - \\
\hline 1522 & 1509 & $\alpha$-Bulnesene & - & - & 0.3 \\
\hline 1530 & 1513 & $\gamma$-Cadinene & 0.1 & 0.8 & 0.2 \\
\hline 1537 & 1522 & $\delta$-Cadinene & 0.5 & 2.9 & 0.5 \\
\hline 1540 & 1528 & Zonarene & - & 0.2 & - \\
\hline 1547 & 1533 & trans-Cadina-1,4-diene & - & 0.2 & - \\
\hline 1552 & 1537 & $\alpha$-Cadinene & - & 0.2 & - \\
\hline 1562 & 1548 & Elemol & 0.2 & - & - \\
\hline 1571 & 1561 & (E)-Nerolidol & - & 0.2 & 1.4 \\
\hline 1577 & 1559 & Germacrene B & 0.2 & - & - \\
\hline 1588 & 1567 & Palustrol & - & - & 0.4 \\
\hline 1599 & 1577 & Spathulenol & 0.9 & 0.6 & 1.0 \\
\hline 1604 & 1592 & Viridiflorol & - & 0.4 & 1.2 \\
\hline 1605 & 1582 & Caryophyllene oxide & 0.6 & 0.4 & - \\
\hline 1612 & 1595 & Cubeban-11-ol & - & 0.6 & - \\
\hline 1615 & 1600 & Guaiol & 0.3 & - & 1.0 \\
\hline 1621 & 1600 & Rosifoliol & - & 0.2 & 0.3 \\
\hline 1625 & 1602 & Ledol & - & - & 1.1 \\
\hline 1632 & 1608 & Humulene epoxide II & - & 0.2 & 0.2 \\
\hline 1642 & 1637 & 5-Guaiene-11-ol & - & - & 0.2 \\
\hline 1658 & 1640 & epi- $\alpha$-Muurolol & - & 0.2 & - \\
\hline 1659 & 1638 & epi- $\alpha$-Cadinol & - & 0.2 & - \\
\hline 1670 & 1652 & $\alpha$-Eudesmol & 0.1 & - & - \\
\hline 1673 & 1652 & $\alpha$-Cadinol & 0.1 & 0.5 & - \\
\hline 1674 & 1662 & 7-epi- $\alpha$-Eudesmol & 0.3 & - & - \\
\hline 1683 & 1670 & epi- $\beta$-Bisabolol & - & - & 0.1 \\
\hline \multirow[t]{7}{*}{1759} & 1732 & Zerumbone & 0.1 & - & - \\
\hline & & Monoterpene hydrocarbons & 35.2 & 10.4 & 21.9 \\
\hline & & Oxygenated monoterpenoids & 0.3 & 0.5 & 2.2 \\
\hline & & Sesquiterpene hydrocarbons & 60.3 & 84.2 & 68.5 \\
\hline & & Oxygenated sesquiterpenoids & 2.6 & 3.5 & 6.9 \\
\hline & & Others & 0.0 & 0.0 & 0.2 \\
\hline & & Total identified & 98.4 & 98.6 & 99.7 \\
\hline
\end{tabular}

$\mathrm{RI}_{\text {calc }}=$ Retention index determined with respect to a homologous series of $n$-alkanes on a HP-5ms column, $\mathrm{RI}_{\mathrm{db}}=$ Retention index from the databases [35-37], B.e. = Beilschmiedia erythrophloia, B.r. = Beilschmiedia robusta, B.y. = Beilschmiedia yunnanensis. 
The major components in B. erythrophloia essential oil were bicyclogermacrene $(30.5 \%)$, (Z)- $\beta$-ocimene $(26.1 \%)$, and $(E)$-caryophyllene $(18.3 \%)$. While qualitatively similar, there are notable differences between the essential oil from Vietnam in this work and that reported by Su and Ho from Taiwan [17]; the sample from Taiwan was rich in $\alpha$-humulene (21.9\%) compared to that from Vietnam (only 2.6\%), but poor in bicyclogermacrene (1.2\%) compared to that from Vietnam.

Both B. robusta and B. yunnanensis leaf oils were rich in $(E)$-caryophyllene $(22.5 \%$ and $16.2 \%$, respectively), $\alpha$-humulene (13.4\% and 9.9\%), and bicyclogermacrene (8.6\% and $8.4 \%)$. The leaf oil of $B$. robusta had a high concentration of germacrene D (20.3\%), while B. yunnanensis oil was rich in 9-epi-(E)-caryophyllene (21.2\%).

The leaf essential compositions of C. concinna (from two locations), C. impressa, and C. infectoria are listed in Table 3. Sesquiterpene hydrocarbons were abundant in both C. impressa and C. infectoria leaf essential oils, while oxygenated sesquiterpenoids were abundant in C. concinna essential oil from Nam Dong and monoterpene hydrocarbons dominated the leaf oil of $C$. concinna from Pu Hoat.

Table 3. Chemical compositions of the leaf essential oils of Cryptocarya species collected in Vietnam.

\begin{tabular}{|c|c|c|c|c|c|c|}
\hline \multirow{2}{*}{$\mathrm{RI}_{\text {calc }}$} & \multirow{2}{*}{$R I_{d b}$} & \multirow{2}{*}{ Compound } & \multicolumn{4}{|c|}{ Percent Composition } \\
\hline & & & C.c. N.D. & C.c. P.H. & C.im. & C.in. \\
\hline 930 & 927 & $\alpha$-thujene & $\operatorname{tr}$ & 0.1 & - & - \\
\hline 931 & 932 & $\alpha$-Pinene & 8.2 & 26.7 & 4.1 & 0.8 \\
\hline 945 & 948 & $\alpha$-Fenchene & $\operatorname{tr}$ & - & - & - \\
\hline 955 & 953 & Camphene & 0.2 & 0.4 & 0.3 & 0.6 \\
\hline 967 & 961 & Benzaldehyde & - & - & - & 0.1 \\
\hline 970 & 971 & Sabinene & $\operatorname{tr}$ & - & - & - \\
\hline 975 & 978 & $\beta$-Pinene & 9.0 & 31.3 & 2.7 & 0.2 \\
\hline 986 & 989 & Myrcene & 3.9 & 11.1 & 3.9 & - \\
\hline 1010 & 1002 & $\alpha$-Phellandrene & - & - & 2.5 & - \\
\hline 1012 & 1009 & $\delta$-3-Carene & 0.1 & - & 0.2 & - \\
\hline 1027 & 1025 & p-Cymene & 0.1 & - & 0.6 & - \\
\hline 1027 & 1030 & Limonene & 2.0 & 2.8 & 0.9 & 0.2 \\
\hline 1028 & 1031 & $\beta$-Phellandrene & $\operatorname{tr}$ & 0.3 & - & - \\
\hline 1033 & 1034 & (Z)- $\beta$-Ocimene & $\operatorname{tr}$ & 0.6 & 0.3 & - \\
\hline 1043 & 1046 & (E)- $\beta$-Ocimene & 0.2 & 8.8 & 4.0 & - \\
\hline 1063 & 1054 & $\gamma$-Terpinene & - & 0.1 & - & - \\
\hline 1094 & 1086 & Terpinolene & - & 0.1 & 0.4 & - \\
\hline 1096 & 1098 & Perillene & 0.1 & - & - & - \\
\hline 1098 & 1101 & $\alpha$-Pinene oxide & 0.2 & - & - & - \\
\hline 1101 & 1095 & Linalool & - & 1.1 & - & 3.4 \\
\hline 1117 & 1116 & (E)-4,8-Dimethylnona-1,3,7-triene & - & - & 0.7 & - \\
\hline 1137 & 1139 & Nopinone & 0.1 & - & - & - \\
\hline 1139 & 1141 & trans-Pinocarveol & 0.3 & - & - & - \\
\hline 1144 & 1145 & trans-Verbenol & 0.1 & - & - & - \\
\hline 1161 & 1164 & Pinocarvone & 0.1 & - & - & - \\
\hline 1194 & 1195 & Myrtenol & 0.3 & - & - & - \\
\hline 1206 & 1201 & Decanal & - & - & 1.6 & - \\
\hline 1299 & 1300 & Tridecane & - & - & 0.2 & - \\
\hline 1308 & 1305 & Undecanal & - & - & 0.2 & - \\
\hline 1332 & 1335 & $\delta$-Elemene & 0.9 & 0.2 & 0.7 & 5.1 \\
\hline 1344 & 1348 & $\alpha$-Cubebene & 0.2 & - & 0.1 & 0.3 \\
\hline 1366 & 1371 & $\alpha$-Ylangene & 0.4 & - & - & - \\
\hline 1367 & 1356 & Eugenol & - & - & - & 0.1 \\
\hline 1373 & 1375 & $\alpha$-Copaene & 0.5 & - & 0.5 & 0.8 \\
\hline 1381 & 1382 & $\beta$-Bourbonene & 0.2 & - & - & 0.3 \\
\hline 1384 & 1373 & $\alpha$-Ylangene & - & - & - & 0.4 \\
\hline 1385 & 1387 & $\beta$-Cubebene & 0.1 & - & - & - \\
\hline 1387 & 1390 & $\beta$-Elemene & 0.9 & 0.1 & 1.2 & 2.1 \\
\hline
\end{tabular}


Table 3. Cont.

\begin{tabular}{|c|c|c|c|c|c|c|}
\hline \multirow{2}{*}{$\mathrm{RI}_{\text {calc }}$} & \multirow{2}{*}{$\mathrm{RI}_{\mathrm{db}}$} & \multirow{2}{*}{ Compound } & \multicolumn{4}{|c|}{ Percent Composition } \\
\hline & & & C.c. N.D. & C.c. P.H. & C.im. & C.in. \\
\hline 1412 & 1408 & Dodecanal & - & - & 10.8 & - \\
\hline 1417 & 1417 & (E)-Caryophyllene & 12.2 & 5.3 & 10.8 & 1.7 \\
\hline 1419 & 1421 & $(E)$ - $\alpha$-Ionone & $\operatorname{tr}$ & - & - & - \\
\hline 1424 & 1430 & $\gamma$-Maaliene & 0.2 & - & - & - \\
\hline 1427 & 1430 & $\beta$-Copaene & 0.3 & - & - & - \\
\hline 1428 & 1426 & $\alpha$-Gurjunene & - & - & 0.4 & - \\
\hline 1430 & 1432 & trans- $\alpha$-Bergamotene & 1.6 & - & 0.9 & - \\
\hline 1436 & 1438 & Aromadendrene & 1.5 & 0.8 & 1.8 & - \\
\hline 1445 & 1437 & $\beta$-Gurjunene & - & - & - & 0.8 \\
\hline 1449 & 1455 & Valerena-4,7(11)-diene & 0.1 & - & - & - \\
\hline 1453 & 1454 & $\alpha$-Humulene & 1.5 & 0.6 & 6.3 & 1.9 \\
\hline 1453 & 1442 & $\alpha$-Maaliene & - & - & 0.2 & - \\
\hline 1456 & 1447 & Guaia-6,9-diene & - & - & - & 0.6 \\
\hline 1457 & 1458 & allo-Aromadendrene & 0.1 & - & - & - \\
\hline 1459 & 1454 & Selina-5,11-diene & - & - & 0.2 & - \\
\hline 1463 & 1463 & cis-Muurola-4(14),5-diene & - & - & 0.1 & - \\
\hline 1466 & 1454 & cis-Muurola-3,5-diene & - & - & - & 0.2 \\
\hline 1472 & 1475 & $\gamma$-Muurolene & 1.6 & 0.4 & 0.7 & 1.3 \\
\hline 1476 & 1482 & $\alpha$-Amorphene & 0.2 & - & 0.7 & 0.7 \\
\hline 1478 & 1480 & Germacrene D & 0.2 & 1.3 & 2.5 & 55.5 \\
\hline 1479 & 1470 & 9-epi-(E)-caryophyllene & - & 0.2 & 0.6 & 0.3 \\
\hline 1480 & 1477 & trans-Cadina-1(6),4-diene & - & - & - & 0.3 \\
\hline 1481 & 1478 & $\gamma$-Gurjunene & 0.1 & - & - & - \\
\hline 1486 & 1489 & $\beta$-Selinene & 0.5 & - & 0.5 & - \\
\hline 1488 & 1491 & Viridiflorene & 0.1 & - & - & - \\
\hline 1489 & 1490 & $\gamma$-Amorphene & 0.3 & - & - & - \\
\hline 1493 & 1497 & $\alpha$-Selinene & 0.6 & - & - & - \\
\hline 1495 & 1497 & $\alpha$-Muurolene & 0.4 & - & - & - \\
\hline 1504 & 1508 & $\beta$-Bisabolene & 0.2 & - & - & - \\
\hline 1505 & 1497 & $\delta$-Selinene & - & - & - & 0.7 \\
\hline 1509 & 1496 & $\gamma$-Amorphene & - & - & - & 0.3 \\
\hline 1512 & 1515 & Cubebol & 0.2 & - & - & - \\
\hline 1512 & 1517 & $(E, E)-\alpha$-Farnesene & - & - & 7.9 & - \\
\hline 1514 & 1511 & Bicyclogermacrene & - & - & 18.7 & 11.4 \\
\hline 1515 & 1518 & $\delta$-Cadinene & 0.7 & 0.7 & 1.1 & - \\
\hline 1518 & 1519 & trans-Calamenene & 0.3 & - & - & - \\
\hline 1520 & 1512 & $\gamma$-Cadinene & 1.3 & 0.4 & 0.3 & - \\
\hline 1521 & 1515 & $\delta$-Amorphene & - & - & 0.2 & 0.7 \\
\hline 1534 & 1538 & $\alpha$-Cadinene & 0.2 & - & - & 0.2 \\
\hline 1538 & 1541 & $\alpha$-Calacorene & 0.4 & - & - & - \\
\hline 1538 & 1531 & cis-Calamene & - & - & - & 0.2 \\
\hline 1546 & 1549 & $\alpha$-Elemol & 0.1 & - & - & 0.2 \\
\hline 1547 & 1540 & trans-Cadina-1,4-diene & - & - & - & 0.1 \\
\hline 1548 & 1551 & Isocaryphyllene oxide & 0.9 & - & - & - \\
\hline 1556 & 1560 & Germacrene B & 0.1 & - & 0.7 & 0.6 \\
\hline 1558 & 1560 & (E)-Nerolidol & 0.2 & - & 0.7 & 0.1 \\
\hline 1559 & 1560 & $\beta$-Calacorene & 0.3 & - & - & - \\
\hline 1560 & 1551 & Selina-3,7(11)-diene & - & - & 0.5 & - \\
\hline 1565 & 1566 & 1,5-Epoxysalvial-4(14)-ene & 0.6 & - & - & - \\
\hline 1575 & 1578 & Spathulenol & 12.3 & 1.1 & 1.4 & 0.1 \\
\hline 1580 & 1587 & Caryophyllene oxide & 21.2 & 0.4 & 0.4 & 0.2 \\
\hline 1583 & 1590 & Globulol & 0.7 & - & - & - \\
\hline 1583 & 1579 & Dendrolasin & - & - & 0.2 & - \\
\hline 1594 & 1593 & Scapanol & - & - & - & 0.2 \\
\hline
\end{tabular}


Table 3. Cont.

\begin{tabular}{|c|c|c|c|c|c|c|}
\hline \multirow{2}{*}{$\mathrm{RI}_{\text {calc }}$} & \multirow{2}{*}{$\mathbf{R I}_{\mathrm{db}}$} & \multirow{2}{*}{ Compound } & \multicolumn{4}{|c|}{ Percent Composition } \\
\hline & & & C.c. N.D. & C.c. P.H. & C.im. & C.in. \\
\hline 1597 & 1594 & Viridiflorol & 0.4 & - & 0.4 & - \\
\hline 1604 & 1612 & 5-epi-7-epi- $\beta$-Eudesmol & 0.2 & - & - & - \\
\hline 1607 & 1613 & Humulene epoxide II & 1.5 & - & 0.3 & - \\
\hline 1612 & 1601 & Cubeban-11-ol & - & - & 0.4 & - \\
\hline 1614 & 1611 & Tetradecanal & - & - & 1.0 & - \\
\hline 1621 & 1615 & Rosifoliol & - & - & 0.4 & - \\
\hline 1623 & 1624 & Muurola-4,10(14)-dien-1 $\beta$-ol & 0.2 & - & - & - \\
\hline 1625 & 1629 & iso-Spathulenol & 1.8 & - & - & - \\
\hline 1630 & 1630 & Caryophylla-4(12),8(13)-dien-5 $\alpha$-ol & 0.9 & - & - & - \\
\hline 1634 & 1636 & Caryophylla-4(12),8(13)-dien-5 $\beta$-ol & 0.6 & - & - & - \\
\hline 1639 & 1640 & $\tau$-Cadinol & 0.2 & 0.4 & - & 0.3 \\
\hline 1641 & 1644 & $\tau$-Muurolol & 0.2 & - & - & 0.2 \\
\hline 1642 & 1637 & 5-Guaien-11-ol & - & - & 0.2 & - \\
\hline 1644 & 1645 & $\delta$-Cadinol & 0.4 & - & - & - \\
\hline 1646 & 1635 & Muurola-4,10(14)-dien-1 $\beta$-ol & - & - & - & 0.1 \\
\hline 1652 & 1656 & $\beta$-Eudesmol & 0.7 & - & - & - \\
\hline 1653 & 1655 & $\alpha-$-Cadinol & 0.9 & - & 0.1 & 0.4 \\
\hline 1654 & 1661 & cis-Calamenen-10-ol & 0.4 & - & - & - \\
\hline 1656 & 1660 & Selin-11-en- $4 \alpha$-ol & 0.3 & - & - & - \\
\hline 1662 & 1662 & 9-Methoxycalamenene & 0.4 & - & - & - \\
\hline 1668 & 1666 & 14-Hydroxy-9-epi-(E)-Caryophyllene & 0.8 & - & - & - \\
\hline 1955 & 1958 & Palmitic acid & 0.2 & - & - & - \\
\hline \multirow[t]{8}{*}{2116} & 2114 & Phytol & - & - & 0.4 & - \\
\hline & & Monoterpene hydrocarbons & 23.7 & 82.3 & 19.9 & 1.8 \\
\hline & & Oxygenated monoterpenoids & 1.1 & 1.1 & 0.0 & 3.4 \\
\hline & & Sesquiterpene hydrocarbons & 28.1 & 10.0 & 57.6 & 86.5 \\
\hline & & Oxygenated sesquiterpenoids & 46.1 & 1.9 & 4.5 & 1.8 \\
\hline & & Diterpenoids & 0.0 & 0.0 & 0.4 & 0.0 \\
\hline & & Others & 0.3 & 0.0 & 14.5 & 0.2 \\
\hline & & Total Identified & 99.2 & 95.3 & 96.9 & 93.7 \\
\hline
\end{tabular}

$\mathrm{RI}_{\text {calc }}=$ Retention index determined with respect to a homologous series of $n$-alkanes on a HP-5ms column, $\mathrm{RI}_{\mathrm{db}}=$ Retention index from the databases [35-37], C.c. N.D. = Cryptocarya concinna from Nam Dong, C.c. P.H. = Cryptocarya concinna from Pu Hoat, C.im. = Cryptocarya impressa, C.in. = Cryptocarya infectoria, $\operatorname{tr}=$ trace.

The leaf essential oils of $C$. concinna from two different collection sites were qualitatively similar, but quantitatively different. That is, the abundant components in the Nam Dong sample were also observed in the $\mathrm{Pu}$ Hoat sample, and vice versa. Thus, for example, $\alpha$-pinene, $\beta$-pinene, and myrcene were abundant in the Pu Hoat sample $(26.7 \%, 31.3 \%$, and $11.1 \%$, respectively) but were found in lower concentrations in the sample from Nam Dong $(8.2 \%, 9.0 \%$, and 3.9\%). Conversely, the sesquiterpenoids, (E)-caryophyllene, spathulenol, and caryophyllene oxide were abundant in the sample from Nam Dong $(12.2 \%, 12.3 \%$, and $21.2 \%$, respectively), but less concentrated in the Pu Hoat sample $(5.3 \%, 1.1 \%$, and $0.4 \%)$.

The major components of the leaf essential oil of $C$. impressa were bicyclogermacrene $(18.7 \%)$, (E)-caryophyllene $(10.8 \%)$, dodecanal $(10.8 \%),(E, E)$ - $\alpha$-farnesene $(7.9 \%)$, and $\alpha$-humulene $(6.3 \%)$. Germacrene D (55.5\%) dominated the essential oil composition of C. infectoria, which was also composed of bicyclogermacrene $(11.4 \%)$ and $\delta$-elemene $(5.1 \%)$ as major components.

The chemical compositions of the leaf essential oils of L. viridis, M. balansae, M. grandifolia, N. ellisoidea, and P. angustifolia are compiled in Table 4. 
Table 4. Chemical compositions of the leaf essential oils of Litsea viridis, Machilus balansae, Machilus grandifolia, Neolitsea ellipsoidea, and Phoebe angustifolia collected in Vietnam.

\begin{tabular}{|c|c|c|c|c|c|c|c|}
\hline \multirow{2}{*}{$\mathbf{R I}_{\text {calc }}$} & \multirow{2}{*}{$\mathbf{R I}_{\mathrm{db}}$} & \multirow{2}{*}{ Compound } & \multicolumn{5}{|c|}{ Percent Composition } \\
\hline & & & L.v. & M.b. & M.g. & N.e. & P.a. \\
\hline 921 & 923 & Tricyclene & - & - & - & - & 0.1 \\
\hline 923 & 927 & $\alpha$-Thujene & - & - & - & - & 0.1 \\
\hline 934 & 933 & $\alpha$-Pinene & 11.1 & 4.4 & 0.3 & 0.2 & 26.9 \\
\hline 949 & 948 & $\alpha$-Fenchene & 0.1 & - & - & - & 0.1 \\
\hline 950 & 953 & Camphene & 0.7 & 0.3 & 0.3 & - & 6.1 \\
\hline 971 & 972 & Sabinene & - & - & - & - & 0.1 \\
\hline 979 & 978 & $\beta$-Pinene & 8.3 & 1.2 & 0.4 & 0.2 & 20.8 \\
\hline 979 & 978 & 1-Octen-3-ol & - & - & 0.1 & - & - \\
\hline 984 & 984 & 6-Methylhept-5-en-2-one & - & - & - & - & 0.1 \\
\hline 990 & 991 & Myrcene & 0.4 & 0.4 & - & 0.5 & 1.5 \\
\hline 1008 & 1006 & $\alpha$-Phellandrene & 0.1 & - & - & - & 0.1 \\
\hline 1022 & 1014 & $\alpha$-Terpinene & 0.2 & - & - & - & - \\
\hline 1026 & 1025 & $p$-Cymene & 0.2 & - & 1.0 & - & 5.0 \\
\hline 1030 & 1030 & Limonene & 1.8 & 0.4 & 1.3 & 0.4 & 3.1 \\
\hline 1030 & 1031 & $\beta$-Phellandrene & - & - & 0.1 & - & 0.2 \\
\hline 1031 & 1032 & 1,8-Cineole & - & - & 0.1 & - & 0.4 \\
\hline 1034 & 1034 & (Z)- $\beta$-Ocimene & 0.1 & 0.1 & - & 3.7 & 0.3 \\
\hline 1046 & 1046 & (E)- $\beta$-Ocimene & 0.3 & 4.5 & - & 87.6 & 0.1 \\
\hline 1063 & 1054 & $\gamma$-Terpinene & 0.5 & - & - & - & - \\
\hline 1069 & 1069 & cis-Linalool oxide (furanoid) & - & - & 0.4 & - & - \\
\hline 1086 & 1086 & trans-Linalool oxide (furanoid) & - & - & 0.4 & - & - \\
\hline 1089 & 1086 & Terpinolene & 0.4 & - & - & - & $\operatorname{tr}$ \\
\hline 1090 & 1093 & $p$-Cymenene & - & - & - & - & 0.1 \\
\hline 1099 & 1101 & $\alpha$-Pinene oxide & - & - & - & - & 0.1 \\
\hline 1100 & 1101 & Linalool & - & - & 3.3 & 1.3 & 0.1 \\
\hline 1105 & 1100 & Nonanal & 0.2 & 0.2 & - & - & - \\
\hline 1117 & 1113 & (E)-4,8-Dimethylnona-1,3,7-triene & 0.4 & 0.4 & - & - & - \\
\hline 1119 & 1119 & endo-Fenchol & - & - & 0.1 & - & 0.1 \\
\hline 1124 & 1124 & cis-p-Menth-2-en-1-ol & - & - & 0.1 & - & - \\
\hline 1141 & 1141 & trans-Pinocarveol & - & - & 0.1 & - & 0.3 \\
\hline 1142 & 1142 & trans- $p$-Menth-2-en-1-ol & - & - & 0.1 & - & - \\
\hline 1143 & 1140 & (E)-Myroxide & - & - & - & 0.2 & - \\
\hline 1145 & 1145 & trans-Verbenol & - & - & - & - & 0.1 \\
\hline 1155 & 1156 & Camphene hydrate & - & - & 0.1 & - & 0.1 \\
\hline 1163 & 1164 & Pinocarvone & - & - & - & - & 0.1 \\
\hline 1172 & 1173 & Borneol & - & - & 0.1 & - & 0.5 \\
\hline 1183 & 1184 & Terpinen-4-ol & 0.2 & - & 0.1 & - & 0.1 \\
\hline 1186 & 1184 & (3Z)-Hexenyl butanoate & - & - & - & 0.3 & - \\
\hline 1188 & 1187 & Cryptone & - & - & 0.5 & - & - \\
\hline 1196 & 1195 & $\alpha$-Terpineol & - & - & 0.3 & - & 0.6 \\
\hline 1206 & 1208 & Decanal & 14.4 & - & - & - & 0.1 \\
\hline 1220 & 1223 & trans-Carveol & - & - & 0.1 & - & - \\
\hline 1245 & 1246 & Carvone & - & - & 0.1 & - & - \\
\hline 1275 & 1275 & trans-Ascaridol glycol & - & - & 0.1 & - & - \\
\hline 1283 & 1285 & Bornyl acetate & - & - & - & - & 1.4 \\
\hline 1299 & 1300 & Tridecane & - & 0.3 & - & - & - \\
\hline 1330 & 1328 & iso-Dihydro carvyl acetate & - & - & - & - & 0.2 \\
\hline 1348 & 1335 & $\delta$-Elemene & - & 1.7 & - & - & - \\
\hline 1358 & 1361 & Neryl acetate & - & - & - & - & 0.1 \\
\hline 1360 & 1345 & $\alpha$-Cubebene & - & 0.1 & - & - & - \\
\hline 1367 & 1367 & Cyclosativene & - & - & 0.2 & - & - \\
\hline
\end{tabular}


Table 4. Cont.

\begin{tabular}{|c|c|c|c|c|c|c|c|}
\hline \multirow{2}{*}{$\mathbf{R I}_{\text {calc }}$} & \multirow{2}{*}{$\mathbf{R I}_{\mathrm{db}}$} & \multirow{2}{*}{ Compound } & \multicolumn{5}{|c|}{ Percent Composition } \\
\hline & & & L.v. & M.b. & M.g. & N.e. & P.a. \\
\hline 1377 & 1378 & Geranyl acetate & - & - & 0.1 & - & - \\
\hline 1377 & 1372 & iso-Ledene & 0.3 & - & - & - & 0.1 \\
\hline 1378 & 1375 & $\alpha$-Copaene & 0.6 & 0.5 & 1.7 & - & 0.3 \\
\hline 1390 & 1379 & Methyl $(E)$-cinnamate & 1.5 & - & - & - & - \\
\hline 1395 & 1390 & $\beta$-Elemene & 1.9 & 1.0 & - & 0.3 & 0.1 \\
\hline 1404 & 1406 & $\alpha$-Gurjunene & - & - & 0.1 & - & 0.2 \\
\hline 1412 & 1412 & Dodecanal & 2.0 & - & - & - & - \\
\hline 1418 & 1416 & cis- $\alpha$-Bergamotene & 0.6 & - & - & - & 0.2 \\
\hline 1424 & 1417 & (E)-Caryophyllene & 0.3 & 8.5 & 0.1 & 0.4 & 5.3 \\
\hline 1425 & 1430 & $\gamma$-Maaliene & - & - & - & - & 0.1 \\
\hline 1428 & 1422 & $\alpha$-Gurjunene & - & 0.5 & - & - & - \\
\hline 1434 & 1432 & $\beta$-Copaene & - & 0.2 & - & - & - \\
\hline 1436 & 1434 & $\beta$-Gurjunene (= Calarene) & - & 0.6 & - & - & 0.1 \\
\hline 1438 & 1432 & trans- $\alpha$-Bergamotene & 0.6 & 0.6 & - & - & 0.8 \\
\hline 1442 & 1438 & $\alpha$-Maaliene & - & 0.3 & - & - & 0.1 \\
\hline 1443 & 1438 & Aromadendrene & 3.0 & 4.5 & 1.0 & 0.3 & 1.8 \\
\hline 1444 & 1445 & Selina-5,11-diene & - & - & 0.1 & - & 0.2 \\
\hline 1445 & 1445 & epi- $\beta$-Santalene & - & - & - & - & 0.1 \\
\hline 1445 & 1437 & $\gamma$-Elemene & 1.0 & - & - & - & - \\
\hline 1453 & 1453 & cis-Muurola-3,5-diene & 0.2 & 0.2 & - & - & - \\
\hline 1454 & 1454 & $\alpha$-Humulene & 0.9 & 1.4 & - & - & 0.6 \\
\hline 1455 & 1452 & $(E)$ - $\beta$-Farnesene & 0.3 & - & - & - & 0.3 \\
\hline 1457 & 1459 & $\beta$-Santalene & - & - & - & - & 0.3 \\
\hline 1458 & 1458 & allo-Aromadendrene & - & - & 0.2 & - & 0.1 \\
\hline 1466 & 1467 & trans-Muurola-3,5-diene & - & 0.5 & - & - & - \\
\hline 1471 & 1476 & $\gamma$-Gurjunene & - & - & - & - & 0.1 \\
\hline 1471 & 1476 & Selina-4,11-diene & - & - & 0.4 & - & - \\
\hline 1479 & 1478 & $\gamma$-Muurolene & 0.5 & 0.8 & 0.6 & 0.1 & 0.1 \\
\hline 1479 & 1474 & 9-epi-(E)-caryophyllene & 0.8 & 0.8 & - & - & - \\
\hline 1482 & 1483 & trans- $\beta$-Bergamotene & - & - & - & - & 0.2 \\
\hline 1485 & 1482 & ar-Curcumene & 0.4 & - & - & - & $\operatorname{tr}$ \\
\hline 1486 & 1482 & $\alpha$-Amorphene & - & 0.6 & 0.1 & - & - \\
\hline 1488 & 1484 & $\gamma$-Curcumene & 0.4 & - & - & - & - \\
\hline 1489 & 1491 & Viridiflorene & 0.2 & - & - & - & 1.2 \\
\hline 1493 & 1487 & $\beta$-Selinene & 1.2 & 0.4 & 2.7 & 0.6 & 0.1 \\
\hline 1497 & 1497 & $\alpha$-Muurolene & - & - & 0.5 & - & 0.1 \\
\hline 1498 & 1501 & (Z)- $\alpha$-Bisabolene & - & - & - & - & 0.1 \\
\hline 1498 & 1496 & Germacrene D & 1.0 & 3.1 & - & 0.1 & - \\
\hline 1503 & 1497 & Bicyclogermacrene & 25.5 & 41.5 & - & - & 1.3 \\
\hline 1503 & 1497 & $\alpha$-Selinene & - & - & 1.3 & 0.5 & - \\
\hline 1505 & 1500 & $\delta$-Selinene & 0.9 & 0.5 & - & - & - \\
\hline 1506 & 1508 & $\beta$-Bisabolene & - & - & - & - & 0.3 \\
\hline 1508 & 1511 & (Z)- $\gamma$-Bisabolene & - & - & - & - & 0.1 \\
\hline 1512 & 1511 & $(E, E)-\alpha$-Farnesene & - & 1.8 & - & - & - \\
\hline 1517 & 1512 & $\gamma$-Cadinene & 0.3 & 0.3 & 0.5 & - & 0.2 \\
\hline 1520 & 1519 & trans-Calamenene & - & - & 0.8 & - & 0.1 \\
\hline 1520 & 1517 & $\beta$-Curcumene & 0.5 & - & - & - & - \\
\hline 1521 & 1516 & $\delta$-Amorphene & 0.2 & 0.3 & - & - & - \\
\hline 1523 & 1518 & $\delta$-Cadinene & 0.9 & 0.7 & 0.2 & 0.2 & 0.2 \\
\hline 1542 & 1531 & $(E)-\gamma$-Bisabolene & 1.0 & - & - & - & - \\
\hline 1547 & 1551 & Elemicin & - & - & 1.2 & - & - \\
\hline 1550 & 1547 & $(E)$ - $\alpha$-Bisabolene & 0.4 & - & - & - & - \\
\hline
\end{tabular}


Table 4. Cont.

\begin{tabular}{|c|c|c|c|c|c|c|c|}
\hline \multirow{2}{*}{$\mathrm{RI}_{\text {calc }}$} & \multirow{2}{*}{$\mathbf{R} \mathbf{I}_{\mathrm{db}}$} & \multirow{2}{*}{ Compound } & \multicolumn{5}{|c|}{ Percent Composition } \\
\hline & & & L.v. & M.b. & M.g. & N.e. & P.a. \\
\hline 1555 & 1555 & (Z)-Dihydronerolidol & - & - & 2.4 & - & - \\
\hline 1560 & 1545 & Selina-3,7(11)-diene & 0.4 & - & - & - & - \\
\hline 1564 & 1561 & (E)-Nerolidol & 1.1 & 8.7 & 22.7 & - & 3.9 \\
\hline 1568 & 1568 & Maaliol & - & - & - & - & 0.1 \\
\hline 1569 & 1568 & Palustrol & - & - & - & - & 0.1 \\
\hline 1569 & 1570 & (E)-Dihydronerolidol & - & - & 2.8 & - & - \\
\hline 1575 & 1575 & Caryolan-8-ol & - & - & 0.8 & - & - \\
\hline 1577 & 1568 & Germacrene B & 1.3 & 0.8 & - & - & - \\
\hline 1578 & 1578 & Spathulenol & 0.9 & 0.6 & - & 0.6 & 5.4 \\
\hline 1585 & 1590 & Globulol & - & - & 10.2 & - & 1.7 \\
\hline 1589 & 1587 & Caryophyllene oxide & - & - & 3.7 & 0.1 & 1.5 \\
\hline 1596 & 1594 & Viridiflorol & 0.4 & 1.6 & 0.7 & - & 0.7 \\
\hline 1601 & 1599 & Cubeban-11-ol & - & 1.0 & 0.6 & - & 0.2 \\
\hline 1604 & 1605 & Ledol & - & - & 0.7 & - & 0.1 \\
\hline 1609 & 1613 & Humulene epoxide II & - & - & 1.5 & - & 0.1 \\
\hline 1611 & 1609 & Rosifoliol & 0.4 & 0.4 & 0.2 & - & 0.2 \\
\hline 1615 & 1617 & Guaiol & 0.8 & - & - & - & - \\
\hline 1627 & 1631 & 1-epi-Cubenol & - & - & 0.8 & - & - \\
\hline 1632 & 1629 & iso-Spathulenol & - & - & - & - & 0.4 \\
\hline 1642 & 1637 & 5-Guaien-11-ol & - & 0.5 & - & - & - \\
\hline 1646 & 1645 & $\alpha$-Muurolol (= $\delta$-Cadinol) & - & - & 1.5 & - & 0.1 \\
\hline 1647 & 1643 & $\tau$-Cadinol & - & 0.2 & 0.7 & - & 0.3 \\
\hline 1649 & 1645 & $\tau$-Muurolol & - & 0.1 & 1.3 & - & 0.1 \\
\hline 1655 & 1655 & $\alpha$-Cadinol & 0.5 & 0.4 & 2.9 & - & 0.2 \\
\hline 1658 & 1660 & Selin-11-en- $4 \alpha$-ol & - & 0.3 & 6.7 & - & - \\
\hline 1665 & 1670 & trans-Calamenen-10-ol & - & - & 1.3 & - & - \\
\hline 1671 & 1665 & $\beta$-Eudesmol & 0.6 & - & - & - & - \\
\hline 1674 & 1676 & Mustakone & - & - & 0.6 & - & - \\
\hline 1674 & 1670 & $\alpha$-Eudesmol & 0.3 & - & - & - & - \\
\hline 1683 & 1672 & epi- $\beta$-Bisabolol & 0.2 & - & - & - & - \\
\hline \multirow[t]{7}{*}{1702} & 1701 & 10-nor-Calamenen-10-one & - & - & 0.4 & - & - \\
\hline & & Monoterpene hydrocarbons & 24.2 & 11.3 & 3.3 & 92.6 & 64.5 \\
\hline & & Oxygenated monoterpenoids & 0.2 & 0.0 & 6.2 & 1.5 & 4.3 \\
\hline & & Sesquiterpene hydrocarbons & 45.6 & 72.2 & 10.6 & 2.5 & 14.8 \\
\hline & & Oxygenated sesquiterpenoids & 5.2 & 13.8 & 62.5 & 0.7 & 15.3 \\
\hline & & Others & 18.5 & 0.9 & 1.3 & 0.3 & 0.1 \\
\hline & & Total Identified & 93.7 & 98.2 & 84.0 & 97.6 & 99.1 \\
\hline
\end{tabular}

$\mathrm{RI}_{\mathrm{calc}}=$ Retention index determined with respect to a homologous series of $n$-alkanes on a HP-5ms column, $\mathrm{RI}_{\mathrm{db}}=$ Retention index from the databases [35-37], L.v. = Litsea viridis, M.b. = Machilus balansae, M.g. = Machilus grandifolia, N.e. $=$ Neolitsea ellisoidea, P.a. $=$ Phoebe angustifolia .

The major components in L. viridis leaf essential oil were bicyclogermacrene (25.5\%), decanal (14.4\%), $\alpha$-pinene $(11.1 \%)$, and $\beta$-pinene ( $8.3 \%)$. This is the first report on the essential oil from this plant.

Although M. balansae and M. grandifolia are considered conspecific, the essential oil compositions showed pronounced differences. The leaf oil of M. balansae was dominated by bicyclogermacrene $(41.5 \%)$, which was not detected in the essential oil of M. grandifolia. Likewise, the sesquiterpene alcohols (E)-nerolidol and globulol were abundant constituents in M. grandifolia $(22.7 \%$ and $10.2 \%$, respectively), but (E)-nerolidol was much lower in M. balansae ( $8.7 \%)$ and globulol was not detected. The taxonomy of these two plants deserves closer scrutiny.

The leaf essential oil of $N$. ellipsoidea was dominated by $(E)$ - $\beta$-ocimene $(87.6 \%)$. (E)- $\beta$-Ocimene was also found to be the dominant compound (85.6\%) in the leaf essential oil of $N$. polycarpa from Vietnam [32], and one of the major components in the leaf essential oils of $N$. sericea from Korea (13.3\%) [38], N. variabillima from Taiwan (13.4\%) [39], and N. aciculata from Korea (9.7\%) [40]. In contrast, 
(E)- $\beta$-ocimene was only a minor component in the leaf oils of $N$. australiensis, $N$. brassii, or N. dealbata from Australia [41], and N. pallens from India [42], and was not observed in N. foliosa leaf essential oil from India [43].

The leaf essential oil of P. angustifolia from Pù Hoạt Nature Reserve (northern Vietnam) in this study was rich in $\alpha$-pinene (26.9\%), $\beta$-pinene (20.8\%), spathulenol (5.4\%), (E)-caryophyllene (5.3\%), and $p$-cymene $(5.0 \%)$, which differs markedly from a previous study on the leaf essential oil from Sao La Nature Reserve (central Vietnam). The previous work reported spathulenol (17.0\%), palmitic acid $(13.0 \%)$, sabinene $(6.4 \%)$, bicyclogermacrene $(5.9 \%)$, and artemisia triene $(5.1 \%)$ to be the major components [32]. There is apparently much variation in the volatile components of this plant.

\subsection{Larvicidal Activity}

The 24-h and 48-h larvicidal activities of Lauraceae leaf essential oils from Vietnam are summarized in Tables 5 and 6. Note that several essential oils were not tested due to lack of sufficient essential oil.

Of the Lauraceae essential oils screened for larvicidal activity, N. ellipsoidea showed the greatest activity against $A$ e. aegypti with $24-\mathrm{h}$ and $48-\mathrm{h} \mathrm{LC}_{50}$ values of 6.59 and $4.04 \mu \mathrm{g} / \mathrm{mL}$, respectively. Similar larvicidal activities were observed against $C x$. quinquefasciatus (24-h and 48-h $\mathrm{LC}_{50}=7.47$ and $4.65 \mu \mathrm{g} / \mathrm{mL}$ ) for this essential oil. Interestingly, although the larvicidal activities of C. infectoria leaf essential oil were not as impressive against Ae. aegypti or Ae. albopictus, the essential oil did show much better activity against $C x$. quinquefasciatus $\left(24-\mathrm{h} \mathrm{LC}_{50}=10.8 \mu \mathrm{g} / \mathrm{mL}\right)$, particularly after $48 \mathrm{~h}$ of exposure (48-h $\left.\mathrm{LC}_{50}=0.402 \mu \mathrm{g} / \mathrm{mL}\right)$. Unfortunately, the limited quantities available for several of the essential oils precluded larvicidal screening. However, the larvicidal activity of the untested essential oils will be investigated in future studies.

Table 5. Twenty-four-hour larvicidal activities of Lauraceae leaf essential oils from Vietnam.

\begin{tabular}{|c|c|c|c|c|}
\hline Lauraceae species & $\mathrm{LC}_{50}$ & $\mathrm{LC}_{90}$ & $x^{2}$ & $p$ \\
\hline \multicolumn{5}{|c|}{ Aedes aegypti } \\
\hline Beilschmiedia erythrophloia & n.t. & n.t. & - & - \\
\hline Beilschmiedia robusta & $24.29(22.36-26.76)$ & $35.22(31.70-41.19)$ & 0.1421 & 0.706 \\
\hline Beilschmiedia yunnanensis & n.t. & n.t. & - & - \\
\hline Cryptocarya concinna (Nam Dong) & $32.54(30.21-35.36)$ & $42.94(39.51-47.91)$ & 0.5537 & 0.758 \\
\hline Cryptocarya concinna (Pu Hoat) & $23.01(20.29-25.83)$ & $40.92(36.50-47.77)$ & 9.298 & 0.010 \\
\hline Cryptocarya impressa & n.t. & n.t. & - & - \\
\hline Cryptocarya infectoria & $21.43(18.85-24.29)$ & $41.88(37.16-48.79)$ & 13.58 & 0.004 \\
\hline Litsea viridis & n.t. & n.t. & - & - \\
\hline Machilus balansae & n.t. & n.t. & - & - \\
\hline Machilus grandifolia & $20.23(18.61-21.93)$ & $29.29(26.85-33.10)$ & 0.001037 & 0.999 \\
\hline Neolitsea ellipsoidea & $6.587(1.478-9.219)$ & $14.00(10.88-17.71)$ & 0.000224 & 1.000 \\
\hline Phoebe angustifolia & $24.29(22.36-26.76)$ & $35.22(31.70-41.19)$ & 0.1421 & 0.931 \\
\hline \multicolumn{5}{|c|}{ Aedes albopictus } \\
\hline Beilschmiedia erythrophloia & n.t. & n.t. & - & - \\
\hline Beilschmiedia robusta & n.t. & n.t. & - & - \\
\hline Beilschmiedia yunnanensis & n.t. & n.t. & - & - \\
\hline Cryptocarya concinna (Nam Dong) & $34.21(31.81-37.04)$ & $43.97(40.67-48.59)$ & 4.651 & 0.098 \\
\hline Cryptocarya concinna (Pu Hoat) & n.t. & n.t. & - & - \\
\hline Cryptocarya impressa & n.t. & n.t. & - & - \\
\hline Cryptocarya infectoria & $61.34(56.76-67.52)$ & $81.29(73.86-93.08)$ & 3.000 & 0.223 \\
\hline Litsea viridis & n.t. & n.t. & - & - \\
\hline Machilus balansae & n.t. & n.t. & - & - \\
\hline Machilus grandifolia & $16.48(14.82-18.02)$ & $25.00(22.90-28.16)$ & $1.86 \times 10^{-5}$ & 1.000 \\
\hline Neolitsea ellipsoidea & n.t. & n.t. & - & - \\
\hline Phoebe angustifolia & $40.18(36.12-44.88)$ & $69.56(62.08-80.81)$ & 31.94 & 0.000 \\
\hline
\end{tabular}


Table 5. Cont.

\begin{tabular}{ccccc}
\hline Lauraceae species & LC $_{\mathbf{5 0}}$ & LC $_{\mathbf{9 0}}$ & $\mathbf{\chi}^{\mathbf{2}}$ & $p$ \\
\hline & Culex quinquefasciatus & & - \\
Beilschmiedia erythrophloia & n.t. & n.t. & - & - \\
Beilschmiedia robusta & n.t. & n.t. & - & - \\
Beilschmiedia yunnanensis & n.t. & n.t. & - & 0.758 \\
Cryptocarya concinna (Nam Dong) & $56.28(52.14-62.30)$ & $75.33(67.95-88.18)$ & 0.5537 & - \\
Cryptocarya concinna (Pu Hoat) & n.t. & n.t. & - & - \\
Cryptocarya impressa & n.t. & n.t. & 18.66 & 0.000 \\
Cryptocarya infectoria & $10.82(6.86-14.27)$ & $53.37(41.49-79.45)$ & - & - \\
Litsea viridis & n.t. & n.t. & - & - \\
Machilus balansae & n.t. & n.t. & -1.000 \\
Machilus grandifolia & $13.59(11.51-15.24)$ & $22.48(20.34-25.94)$ & $6.1 \times 10^{-6}$ & 1.931 \\
Neolitsea ellipsoidea & $7.465(3.904-9.956)$ & $19.84(16.52-25.64)$ & 0.1427 & 0.931 \\
Phoebe angustifolia & $20.70(19.36-21.96)$ & $26.60(25.10-28.63)$ & 0.000 & 1.000 \\
\hline
\end{tabular}

n.t. $=$ not tested due to insufficient essential oil.

The major component of $N$. ellipsoidea leaf essential oil, $(E)-\beta$-ocimene $(87.6 \%)$, is not likely responsible for the observed larvicidal activity. The (E)- $\beta$-ocimene-rich (94.8\%) essential oil of Porophyllum ruderale showed an $\mathrm{LC}_{50}$ of $173.7 \mu \mathrm{g} / \mathrm{mL}$ against Ae. aegypti [44]. Likewise, the essential oil of Syzygium jambolana, with (Z)- $\beta$-ocimene (27.2\%) and (E)- $\beta$-ocimene (12.2\%), was inactive against Ae. aegypti $\left(\mathrm{LC}_{50}=433 \mu \mathrm{g} / \mathrm{mL}\right)$ [45]. The excellent larvicidal activity of $N$. ellipsoidea essential oil can likely be attributed to synergistic effects involving minor components.

The leaf essential oil of $C$. infectoria was rich in the germacrene sesquiterpenes germacrene $\mathrm{D}(55.5 \%)$ and bicyclogermacrene (11.4\%), and these compounds may be responsible for the larvicidal activity. Germacrene $\mathrm{D}$ has demonstrated notable larvicidal activity against Ae. aegypti and Cx. quinquefasciatus $\left(\mathrm{LC}_{50}=18.8\right.$ and $21.3 \mu \mathrm{g} / \mathrm{mL}$, respectively) [46], and bicyclogermacrene was larvicidal against Ae. albopictus and $C x$. tritaeniorhynchus $\left(\mathrm{LC}_{50}=11.1\right.$ and $12.5 \mu \mathrm{g} / \mathrm{mL}$, respectively) [47].

Table 6. Forty-eight-hour larvicidal activities of Lauraceae leaf essential oils from Vietnam.

\begin{tabular}{|c|c|c|c|c|}
\hline Lauraceae species & $\mathrm{LC}_{50}$ & $\mathrm{LC}_{90}$ & $x^{2}$ & $p$ \\
\hline \multicolumn{5}{|c|}{ Aedes aegupti } \\
\hline Beilschmiedia erythrophloia & n.t. & n.t. & - & - \\
\hline Beilschmiedia robusta & $22.00(19.81-24.45)$ & $35.64(31.82-41.93)$ & 0.6316 & 0.427 \\
\hline Beilschmiedia yunnanensis & n.t. & n.t. & - & - \\
\hline Cryptocarya concinna (Nam Dong) & $32.03(29.72-34.84)$ & $42.58(39.12-47.64)$ & 0.1879 & 0.910 \\
\hline Cryptocarya concinna (Pu Hoat) & $16.22(12.81-18.90)$ & $33.46(29.37-40.63)$ & 1.028 & 0.598 \\
\hline Cryptocarya impressa & n.t. & n.t. & - & - \\
\hline Cryptocarya infectoria & $18.94(16.39-21.65)$ & $39.12(34.54-45.97)$ & 13.16 & 0.004 \\
\hline Litsea viridis & n.t. & n.t. & - & - \\
\hline Machilus balansae & n.t. & n.t. & - & - \\
\hline Machilus grandifolia & $16.17(14.61-17.64)$ & $24.03(22.07-26.93)$ & $1.4 \times 10^{-6}$ & 1.000 \\
\hline Neolitsea ellipsoidea & $4.038(0.004-7.585)$ & $11.12(2.12-14.74)$ & 0.004798 & 0.998 \\
\hline Phoebe angustifolia & $22.46(20.59-24.69)$ & $33.44(30.10-39.07)$ & 0.06258 & 0.969 \\
\hline \multicolumn{5}{|c|}{ Aedes albopictus } \\
\hline Beilschmiedia erythrophloia & n.t. & n.t. & - & - \\
\hline Beilschmiedia robusta & n.t. & n.t. & - & - \\
\hline Beilschmiedia yunnanensis & n.t. & n.t. & - & - \\
\hline Cryptocarya concinna (Nam Dong) & $30.19(27.92-33.28)$ & $40.26(36.49-46.42)$ & 1.922 & 0.383 \\
\hline Cryptocarya concinna (Pu Hoat) & n.t. & n.t. & - & - \\
\hline Cryptocarya impressa & n.t. & n.t. & - & - \\
\hline Cryptocarya infectoria & $58.80(54.40-64.96)$ & $78.50(71.00-90.93)$ & 1.282 & 0.527 \\
\hline Litsea viridis & n.t. & n.t. & - & - \\
\hline Machilus balansae & n.t. & n.t. & - & - \\
\hline Machilus grandifolia & $15.45(13.62-17.07)$ & $24.47(22.28-27.88)$ & $3.69 \times 10^{-5}$ & 1.000 \\
\hline Neolitsea ellipsoidea & n.t. & n.t. & - & - \\
\hline Phoebe angustifolia & $35.28(31.29-39.67)$ & $64.97(57.71-76.08)$ & 23.97 & 0.000 \\
\hline
\end{tabular}


Table 6. Cont.

\begin{tabular}{ccccc}
\hline Lauraceae species & LC $_{\mathbf{5 0}}$ & LC $_{\mathbf{9 0}}$ & $\boldsymbol{\chi}^{\mathbf{2}}$ & $p$ \\
\hline & Culex quinquefasciatus & & - \\
Beilschmiedia erythrophloia & n.t. & n.t. & - & - \\
Beilschmiedia robusta & n.t. & n.t. & - & - \\
Beilschmiedia yunnanensis & n.t. & n.t. & - & - \\
Cryptocarya concinna (Nam Dong) & $41.89(37.88-46.65)$ & $69.84(62.41-80.77)$ & 5.550 & 0.062 \\
Cryptocarya concinna (Pu Hoat) & n.t. & n.t. & - & - \\
Cryptocarya impressa & n.t. & n.t. & - & - \\
Cryptocarya infectoria & $0.402(0.000-2.947)$ & $11.39(0.04-21.64)$ & 6.397 & 0.041 \\
Litsea viridis & n.t. & n.t. & - & - \\
Machilus balansae & n.t. & n.t. & - & - \\
Machilus grandifolia & $11.56(9.13-13.14)$ & $19.24(17.25-23.07)$ & 0.000 & 1.000 \\
Neolitsea ellipsoidea & $4.650(0.061-7.988)$ & $11.89(4.63-15.36)$ & 0.002409 & 0.999 \\
Phoebe angustifolia & $12.21(8.66-14.46)$ & $24.28(21.55-29.24)$ & 0.002467 & 0.999 \\
\hline & n.t. $=$ not tested due to insufficient essential oil. & &
\end{tabular}

The marginal larvicidal activity of $C$. concinna from Nam Dong is consistent with the marginal activities observed for the major components. (E)-Caryophyllene, caryophyllene oxide, and $\alpha$-pinene have shown modest mosquito larvicidal activities [48]. $\beta$-Pinene, however, has been shown to be more active than $\alpha$-pinene: $(-)-\beta$-pinene $\left(\mathrm{LC}_{50}=65 \mu \mathrm{g} / \mathrm{mL}\right.$ against $C x$. quinquefasciatus) [49], $\left(\mathrm{LC}_{50}=15.4 \mu \mathrm{g} / \mathrm{mL}\right.$ against $A$ e. aegypti) [50]; (+)- $\beta$-pinene $\left(\mathrm{LC}_{50}=22.4 \mu \mathrm{g} / \mathrm{mL}\right.$ against Ae. aegypti) [49]. Spathulenol-rich essential oils have also shown only marginal larvicidal activities. The stem essential oil of Tephrosia toxicaria (42.3\% spathulenol) had an $\mathrm{LC}_{50}$ of $63.1 \mu \mathrm{g} / \mathrm{mL}$ against Ae aegypti [51], while Guarea sylvatica essential oil from branches (14.3\% spathulenol) showed $\mathrm{LC}_{50}$ against Ae. aegypti of $274 \mu \mathrm{g} / \mathrm{mL}$ [52].

\subsection{Antimicrobial Activity}

Several of the leaf essential oils of the Lauraceae were screened for antimicrobial activity (Table 7). All of the essential oils tested showed good antibacterial activities against the Gram-positive organisms. Both L. viridis and N. ellipsoidea leaf essential oils demonstrated particularly notable activities against E. faecalis and B. cereus with minimum inhibitory concentration (MIC) values of $16 \mu \mathrm{g} / \mathrm{mL}$. The leaf essential oil of $C$. impressa also showed excellent anticandidal activity against $C$. albicans with an MIC of $16 \mu \mathrm{g} / \mathrm{mL}$.

The major component of L. viridis leaf oil, bicyclogermacrene, has shown antibacterial activity against $B$. cereus [53]. Likewise, $\beta$-pinene was shown to be active against $E$. faecalis [54] as well as several other Gram-positive organisms [55]. Similarly, $\alpha$-pinene has activity against several Gram-positive bacteria $[55,56]$. Decanal has also exhibited antibacterial activity $[57,58]$. Thus, the major components of $L$. viridis leaf essential oil, bicyclogermacrene, decanal, $\alpha$-pinene, and $\beta$-pinene, can account for the observed antibacterial activity.

(E)- $\beta$-Ocimene dominated the leaf essential oil of $N$. ellipsoidea, but this compound has demonstrated relatively marginal antibacterial activity [55]. Synergistic interactions of (E)- $\beta$-ocimene with minor essential oil components may play a role in the antibacterial activity of $N$. ellipsoidea leaf oil.

The components responsible for the anticandidal activity of $C$. impressa leaf essential oil are not obvious. Neither (E)-caryophyllene nor $\alpha$-humulene have shown strong anti-Candida albicans activity $[54,56]$. The anticandidal activity of bicyclogermacrene itself has apparently not been determined. However, essential oils rich in both bicyclogermacrene and $(E)$-caryophyllene do not exhibit notable activity against Candida spp. [59,60]. Dodecanal, however, has shown activity against $C$. albicans with an MIC of $125 \mu \mathrm{g} / \mathrm{mL}$ [61]. 
Table 7. Antimicrobial activities of leaf essential oils of Lauraceae from Vietnam.

\begin{tabular}{|c|c|c|c|c|c|c|c|}
\hline Sample & $\begin{array}{c}\text { Enterococcus faecalis } \\
\text { ATCC } 299212\end{array}$ & $\begin{array}{c}\text { Gram (+) } \\
\text { Staphylococcus aureus } \\
\text { ATCC } 25923\end{array}$ & $\begin{array}{l}\text { Bacillus cereus } \\
\text { ATCC } 14579\end{array}$ & $\begin{array}{l}\text { Escherichia coli } \\
\text { ATCC } 25922\end{array}$ & $\begin{array}{c}\text { Gram (-) } \\
\text { Pseudomonas aeruginosa } \\
\text { ATCC } 27853\end{array}$ & $\begin{array}{c}\text { Salmonella enterica } \\
\text { ATCC } 13076\end{array}$ & $\begin{array}{c}\text { Yeast } \\
\text { Candida albicans } \\
\text { ATCC } 10231\end{array}$ \\
\hline & & & & $\mathrm{MIC}(\mu \mathrm{g} / \mathrm{mL})$ & & & \\
\hline Beilschmiedia erythrophloia & 32 & 64 & 64 & n.a. & n.a. & n.a. & 128 \\
\hline Beilschmiedia robusta & 64 & 64 & n.a. & 64 & n.a. & n.a. & n.a. \\
\hline Beilschmiedia yunnanensis & 64 & 64 & 64 & n.a. & n.a. & n.a. & 256 \\
\hline $\begin{array}{l}\text { Cryptocarya concinna } \\
\text { (Pu Hoat) }\end{array}$ & 32 & 128 & 64 & n.a. & 128 & 256 & 64 \\
\hline Cryptocarya impressa & 64 & 64 & 128 & 64 & n.a. & n.a. & 16 \\
\hline Cryptocarya infectoria & 128 & 64 & 128 & n.a. & 64 & 128 & 64 \\
\hline Litsea viridis & 16 & 64 & 16 & n.a. & n.a. & n.a. & 128 \\
\hline Machilus balansae & 64 & 128 & 128 & n.a. & n.a. & n.a. & n.a. \\
\hline Neolitsea ellipsoidea & 16 & 32 & 16 & 128 & n.a. & n.a. & 128 \\
\hline Streptomycin & 256 & 256 & 128 & 32 & 256 & 128 & n.t. \\
\hline Nistatin & n.t. & n.t. & n.t. & n.t. & n.t. & n.t. & 8 \\
\hline Cyclohexamide & n.t. & n.t. & n.t. & $\begin{array}{l}\text { n.t. } \\
\mathrm{IC}_{50}(\mu \mathrm{g} / \mathrm{mL})\end{array}$ & n.t. & n.t. & 32 \\
\hline Beilschmiedia erythrophloia & 10.34 & 20.34 & 34.78 & n.a. & n.a. & n.a. & 56.78 \\
\hline Beilschmiedia robusta & 20.76 & 18.67 & n.a. & 17.88 & n.a. & n.a. & n.a. \\
\hline Beilschmiedia yunnanensis & 17.99 & 20.34 & 24.67 & n.a. & n.a. & n.a. & 100.34 \\
\hline $\begin{array}{l}\text { Cryptocarya concinna } \\
\text { (Pu Hoat) }\end{array}$ & 8.99 & 40.67 & 18.99 & n.a. & 48.98 & 145.34 & 25.67 \\
\hline Cryptocarya impressa & 20.34 & 28.77 & 47.67 & 18.78 & n.a. & n.a. & 5.89 \\
\hline Cryptocarya infectoria & 65.33 & 32.67 & 63.56 & n.a. & 33.22 & 65.66 & 32.22 \\
\hline Litsea viridis & 2.45 & 18.99 & 7.67 & n.a. & n.a. & n.a. & 56.78 \\
\hline Machilus balansae & 18.78 & 50.35 & 45.77 & n.a. & n.a. & n.a. & n.a. \\
\hline Neolitsea ellipsoidea & 3.99 & 7.98 & 5.67 & 57.78 & n.a. & n.a. & 56.67 \\
\hline
\end{tabular}




\section{Materials and Methods}

\subsection{Plant Collection}

Leaves were collected from wild-growing trees in north-central Vietnam. Plants were identified by Do Ngoc Dai and voucher specimens (Table 1) have been deposited in the plant specimen room, Faculty Agriculture, Forestry and Fishery, Nghe An, College of Economics. In each case, the fresh leaves were chopped and $2.0 \mathrm{~kg}$ was subjected to hydrodistillation using a Clevenger-type apparatus.

\subsection{Analysis of the Oils}

Gas chromatographic (GC) analysis was performed on an Agilent Technologies HP 7890A Plus Gas chromatograph equipped with a FID and fitted with HP-5ms column $(30 \mathrm{~m} \times 0.25 \mathrm{~mm}$, film thickness $0.25 \mu \mathrm{m}$, Agilent Technologies, Santa Clara, CA, USA). The analytical conditions were: carrier gas $\mathrm{H}_{2}$ ( $1 \mathrm{~mL} / \mathrm{min}$ ), injector temperature (PTV: programmable temperature vaporization) $250{ }^{\circ} \mathrm{C}$, detector temperature $260{ }^{\circ} \mathrm{C}$, column temperature programmed from $60{ }^{\circ} \mathrm{C}$ ( 2 min hold) to $220^{\circ} \mathrm{C}$ ( 10 min hold $)$ at $4{ }^{\circ} \mathrm{C} / \mathrm{min}$. Samples were injected using a split mode with a split ratio of 10:1. The volume injected was $1.0 \mu \mathrm{L}$. Inlet pressure was $6.1 \mathrm{kPa}$.

An Agilent Technologies (Santa Clara, CA, USA) HP 7890A Plus Chromatograph fitted with a fused silica capillary HP-5ms column $(30 \mathrm{~m} \times 0.25 \mathrm{~mm}$, film thickness $0.25 \mu \mathrm{m})$ and interfaced with a mass spectrometer HP 5973 MSD was used for the GC/MS analysis, under the same conditions as those used for GC analysis. The conditions were the same as described above with $\mathrm{He}(1 \mathrm{~mL} / \mathrm{min}) \mathrm{as}$ carrier gas. The MS conditions were as follows: ionization voltage $70 \mathrm{eV}$; emission current $40 \mathrm{~mA}$; acquisitions scan mass range of 35-350 amu at a sampling rate of $1.0 \mathrm{scan} / \mathrm{s}$. Compound identification was carried out by comparison of the MS fragmentation patterns and calculated retention indices with those available in the databases [35-37] and, when available, with standard substances.

\subsection{Mosquito Larvicidal Assays}

Larvicidal activities against Aedes aegypti, Aedes albopictus, and Culex quinquefasciatus were carried out as previously described [62]; $\mathrm{LC}_{50}$ values, $\mathrm{LC}_{90}$ values, and 95\% confidence limits were determined by log-probit analysis using Minitab ${ }^{\circledR} 19$ (Minitab, LLC, State College, PA, USA).

\subsection{Antimicrobial Assays}

The bacterial growth inhibition of the essential oils was evaluated using three strains of Gram-positive test bacteria, Enterococcus faecalis (ATCC299212), Staphylococcus aureus (ATCC25923), Bacillus cereus (ATCC14579), three strains of Gram-negative test bacteria, Escherichia coli (ATCC 25922), Pseudomonas aeruginosa (ATCC27853), Salmonella enterica (ATCC13076) and one strain of yeast, Candida albicans (ATCC 10231). Minimum inhibitory concentration (MIC) and median inhibitory concentration $\left(\mathrm{IC}_{50}\right)$ values were measured by the microdilution broth susceptibility assay as previously described [62].

\section{Conclusions}

Of the eleven species of Lauraceae examined in this work, the leaf essential oil of Neolitsea ellipsoidea, dominated by $(E)$ - $\beta$-ocimene, showed excellent larvicidal activity against Aedes aegypti and antibacterial activity against Enterococcus faecalis and Bacillus cereus; Cryptocarya infectoria leaf essential oil, rich in germacrene D and bicyclogermacrene, showed excellent larvicidal activity on Culex quinquefasciatus and anticandidal activity against Candida albicans. The leaf essential oil of Litsea viridis, which was rich in bicyclogermacrene, also showed good antibacterial properties. The biological properties of these Lauraceae essential oils suggest that they may serve as potential "green" alternatives, as also described for Lamiaceae family plants [63], for use as insect control or antimicrobial agents. 
Author Contributions: Conceptualization, D.N.D. and W.N.S.; methodology, D.N.D., L.T.H., D.T.M.C., N.H.H., I.A.O., W.N.S.; validation, D.N.D. and W.N.S.; formal analysis, L.T.H., W.N.S.; investigation, N.T.C., L.T.H., N.T.Y., D.T.M.C., I.A.O.; resources, D.N.D.; data curation, W.N.S.; writing-original draft preparation, W.N.S., D.N.D.; writing—review and editing, D.N.D., L.T.H., W.N.S.; supervision, D.N.D.; project administration, D.N.D.; funding acquisition, D.N.D. All authors have read and agreed to the published version of the manuscript.

Funding: This research is funded by Vietnam National Foundation for Science and Technology Development (NAFOSTED) under grant number: 106.03-2018.02.

Acknowledgments: W.N.S. participated in this work as part of the activities of the Aromatic Plant Research Center (APRC, https://aromaticplant.org/).

Conflicts of Interest: The authors declare no conflict of interest.

\section{References}

1. Van der Werff, H. A synopsis of the genus Beilschmiedia (Lauraceae) in Madagascar. Adansonia 2003, 25, 77-92.

2. Schroeder, C.A. Some useful plants of the botanical family Lauraceae. Calif. Avocado Soc. Yearb. 1976, 59, 30-34.

3. Krochmal, A. Medicinal plants and Appalachia. Econ. Bot. 1968, 22, 332-337. [CrossRef]

4. Tucker, A.O.; Maciarello, M.J.; Burbage, P.W.; Sturtz, G. Spicebush [Lindera benzoin (L.) Blume var. benzoin, Lauraceae]: A tea, spice, and medicine. Econ. Bot. 1994, 48, 333-336. [CrossRef]

5. Rhind, J.P. Essential Oils: A Comprehensive Handbook for Aromatic Therapy; Singing Dragon: London, UK, 2020; ISBN 978-1787752290.

6. May, P.H.; Barata, L.E.S. Rosewood exploitation in the Brazilian Amazon: Options for sustainable production. Econ. Bot. 2004, 58, 257-265. [CrossRef]

7. Chen, W.; Vermaak, I.; Viljoen, A. Camphor-A fumigant during the black death and a coveted fragrant wood in ancient Egypt and Babylon: A review. Molecules 2013, 18, 5434-5454. [CrossRef] [PubMed]

8. Kong, D.-G.; Zhao, Y.; Li, G.-H.; Chen, B.-J.; Wang, X.-N.; Zhou, H.-L.; Lou, H.-X.; Ren, D.-M.; Shen, T. The genus Litsea in traditional Chinese medicine: An ethnomedical, phytochemical and pharmacological review. J. Ethnopharmacol. 2015, 164, 256-264. [CrossRef] [PubMed]

9. Missouri Botanical Garden Tropicos. Available online: www.tropicos.org (accessed on 14 March 2020).

10. Beilschmiedia Nees. Available online: https://www.discoverlife.org/mp/20q?search=Beilschmiedia (accessed on 16 March 2020).

11. Salleh, W.M.N.H.W.; Ahmad, F.; Yen, K.H.; Zulkifli, R.M. A review on chemical constituents and biological activities of the genus Beilschmiedia (Lauraceae). Trop. J. Pharm. Res. 2015, 14, 2139-2150. [CrossRef]

12. Wu, C.Y.; Raven, P.H.; Hong, D.Y. Flora of China. Available online: http://www.efloras.org/volume_page. aspx?volume_id=2007\&flora_id=2 (accessed on 14 March 2020).

13. Chen, J.-J.; Kuo, W.-L.; Sung, P.-J.; Chen, I.-S.; Cheng, M.-J.; Lim, Y.-P.; Liao, H.-R.; Chang, T.-H.; Wei, D.-C.; Chen, J.-Y. Beilschamide: A new amide, and cytotoxic constituents of Beilschmiedia erythrophloia. Chem. Nat. Compd. 2015, 51, 302-305. [CrossRef]

14. Dao, N.K. Flora of Vietnam, Lauraceae Juss; Publishing House for Science \& Technology: Hanoi, Vietnam, 2017; Volume 17.

15. Yang, P.-S.; Cheng, M.-J.; Chen, J.-J.; Chen, I.-S. Two new endiandric acid analogs, a new benzopyran, and a new benzenoid from the root of Beilschmiedia erythrophloia. Helv. Chim. Acta 2008, 91, 2130-2138. [CrossRef]

16. Yang, P.-S.; Cheng, M.-J.; Peng, C.-F.; Chen, J.-J.; Chen, I.-S. Endiandric acid analogues from the roots of Beilschmiedia erythrophloia. J. Nat. Prod. 2009, 72, 53-58. [CrossRef] [PubMed]

17. Su, Y.-C.; Ho, C.-L. Composition and in-vitro cytotoxic activities of the leaf essential oil of Beilschmiedia erythrophloia from Taiwan. Nat. Prod. Commun. 2013, 8, 143-144. [PubMed]

18. Allen, C.K. Studies in the Lauraceae, V: Some eastern Asiatic species of Beilschmiedia and related genera. J. Arnold Arbor. 1942, 23, 444-463. [CrossRef]

19. Mabberley, D.J. Mabberley's Plant-Book, 3rd ed.; Cambridge University Press: Cambridge, UK, 2008.

20. Chang, H.-S.; Tang, J.-Y.; Yen, C.-Y.; Huang, H.-W.; Wu, C.-Y.; Chung, Y.-A.; Wang, H.-R.; Chen, I.-S.; Huang, M.-Y.; Chang, H.-W. Antiproliferation of Cryptocarya concinna-derived cryptocaryone against oral cancer cells involving apoptosis, oxidative stress, and DNA damage. BMC Complement. Altern. Med. 2016, 16, 94. [CrossRef] 
21. Yang, B.-Y.; Shi, Y.-M.; Luo, J.-G.; Kong, L.-Y. Two new arylalkenyl $\alpha, \beta$-unsaturated $\delta$-lactones with cytotoxic activity from the leaves and twigs of Cryptocarya concinna. Nat. Prod. Res. 2017, 31, 1409-1413. [CrossRef]

22. Huang, W.; Zhang, W.-J.; Cheng, Y.-Q.; Jiang, R.; Wei, W.; Chen, C.-J.; Wang, G.; Jiao, R.-H.; Tan, R.-X.; Ge, H.-M. Cytotoxic and antimicrobial flavonoids from Cryptocarya concinna. Planta Med. 2014, 80, 925-930. [CrossRef]

23. Kew Science Cryptocarya impressa Miq. Available online: http://powo.science.kew.org/taxon/urn:lsid: ipni.org:names:463940-1\#source-KBD (accessed on 16 March 2020).

24. De Kok, R.P.J. A revision of Cryptocarya (Lauraceae) from Thailand and Indochina. Gard. Bull. Singapore 2015, 67, 309-350. [CrossRef]

25. De Kok, R.P.J. A revision of Cryptocarya R. Br. (Lauraceae) of Peninsular Malaysia. Kew Bull. $2016,71,7$. [CrossRef]

26. De Kok, R.P.J. Two new records of Litsea (Lauraceae) from Singapore and the lectotypification of twenty-two names from several Lauraceae genera. Gard. Bull. Singapore 2017, 69, 167-177. [CrossRef]

27. Chen, Y.-C.; Chen, I.-S.; Guh, J.-H. Cryptocaryone, isolated from Cryptocarya infectoria, induces apoptosis through extrinsic pathways: The involvement of death receptor clustering and FADD/caspase-8 activation cascades. Clin. Cancer Res. 2007, 13, C42.

28. Dumontet, V.; Gaspard, C.; Van Hung, N.; Fahy, J.; Tchertanov, L.; Sévenet, T.; Guéritte, F. New cytotoxic flavonoids from Cryptocarya infectoria. Tetrahedron 2001, 57, 6189-6196. [CrossRef]

29. Othman, W.N.N.W.; Liew, S.Y.; Khaw, K.Y.; Murugaiyah, V.; Litaudon, M.; Awang, K. Cholinesterase inhibitory activity of isoquinoline alkaloids from three Cryptocarya species (Lauraceae). Bioorganic Med. Chem. 2016, 24, 4464-4469. [CrossRef] [PubMed]

30. Tang, S.-C.; Xu, W.-B.; Wei, F.-N. Machilus parapauhoi sp. nov. and a new synonym of Machilus (Lauraceae) from east Asia. Nord. J. Bot. 2010, 28, 503-505. [CrossRef]

31. Allen, C.K. Studies in the Lauraceae. I. Chinese and Indo-Chinese species of Litsea, Neolitsea, and Actinodaphne. Ann. Missouri Bot. Gard. 1938, 25, 361-434. [CrossRef]

32. Thang, T.D.; Dai, D.N.; Thai, T.H.; Ogunwande, I.A. Essential oils of Phoebe angustifolia Meisn., Machilus velutina Champ. ex Benth. and Neolitsea polycarpa Liou (Lauraceae) from Vietnam. Rec. Nat. Prod. 2013, 7, 192-200.

33. Salleh, W.M.N.H.W.; Ahmad, F.; Khong, H.Y.; Zulkifli, R.M. Comparative study of the essential oils of three Beilschmiedia species and their biological activities. Int. J. Food Sci. Technol. 2016, 51, 240-249. [CrossRef]

34. Setzer, W.N.; Haber, W.A. Leaf essential oil composition of five species of Beilschmiedia from Monteverde, Costa Rica. Nat. Prod. Commun. 2007, 2, 79-83. [CrossRef]

35. Mondello, L. FFNSC 3; Shimadzu Scientific Instruments: Columbia, MD, USA, 2016.

36. NIST17; National Institute of Standards and Technology: Gaithersburg, MD, USA, 2017.

37. Adams, R.P. Identification of Essential Oil Components by Gas Chromatography/Mass Spectrometry, 4th ed.; Allured Publishing: Carol Stream, IL, USA, 2007.

38. Yoon, W.-J.; Moon, J.-Y.; Kang, J.-Y.; Kim, G.-O.; Lee, N.H.; Hyun, C.-G. Neolitsea sericea essential oil attenuates LPS-induced inflammation in RAW 264.7 macrophages by suppressing NF-kB and MAPK activation. Nat. Prod. Commun. 2010, 5, 1311-1316. [CrossRef]

39. Su, Y.-C.; Hsu, K.-P.; Wang, E.I.-C.; Ho, C.-L. Composition and in vitro anticancer activities of the leaf essential oil of Neolitsea variabillima from Taiwan. Nat. Prod. Commun. 2013, 8, 531-532. [CrossRef]

40. Jeong, M.-J.; Yang, J.; Choi, W.-S.; Kim, J.-W.; Kim, S.J.; Park, M.-J. Chemical compositions and antioxidant activities of essential oil extracted from Neolitsea aciculata (Blume) Koidz leaves. J. Korean Wood Sci. Technol. 2017, 45, 96-106. [CrossRef]

41. Brophy, J.J.; Goldsack, R.J.; Fookes, C.J.R.; Forster, P.I. The leaf oils of the Australian species of Neolitsea (Lauraceae). J. Essent. Oil Res. 2002, 14, 191-195. [CrossRef]

42. Padalia, R.C.; Chanotiya, C.S.; Thakuri, B.C.; Mathela, C.S. Germacranolide rich essential oil from Neolitsea pallens. Nat. Prod. Commun. 2007, 2, 591-593. [CrossRef]

43. John, A.J.; Karunakaran, V.P.; George, V.; Pradeep, N.S.; Sethuraman, M.G. Chemical composition and antibacterial activity of leaf oil of Neolitsea foliosa (Nees) Gamble var. caesia (Meisner) Gamble. J. Essent. Oil Res. 2007, 19, 498-500. [CrossRef] 
44. Fontes, U.R., Jr.; Ramos, C.S.; Serafini, M.R.; Cavalcanti, S.C.H.; Alves, P.B.; Lima, G.M.; Andrade, P.H.S.; Bonjardim, L.R.; Quintans, L.J., Jr.; Araújo, A.S. Evaluation of the lethality of Porophyllum ruderale essential oil against Biomphalaria glabrata, Aedes aegypti and Artemia salina. African J. Biotechnol. 2012, 11, 3169-3172.

45. Cavalcanti, E.S.B.; de Morais, S.M.; Lima, M.A.A.; Santana, E.W.P. Larvicidal activity of essential oils from Brazilian plants against Aedes aegypti L. Mem. Inst. Oswaldo Cruz 2004, 99, 541-544. [CrossRef] [PubMed]

46. Govindarajan, M. Chemical composition and larvicidal activity of leaf essential oil from Clausena anisata (Willd.) Hook. f. ex Benth (Rutaceae) against three mosquito species. Asian Pac. J. Trop. Med. 2010, 3, 874-877. [CrossRef]

47. Govindarajan, M.; Benelli, G. Ecotoxicology and environmental safety eco-friendly larvicides from Indian plants: Effectiveness of lavandulyl acetate and bicyclogermacrene on malaria, dengue and Japanese encephalitis mosquito vectors. Ecotoxicol. Environ. Saf. 2016, 133, 395-402. [CrossRef]

48. Hung, N.H.; Satyal, P.; Hieu, H.V.; Chuong, N.T.H.; Dai, D.N.; Huong, L.T.; Tai, T.A.; Setzer, W.N. Mosquito larvicidal activity of the essential oils of Erechtites species growing wild in Vietnam. Insects 2019, 10, 47. [CrossRef]

49. Pavela, R. Acute toxicity and synergistic and antagonistic effects of the aromatic compounds of some essential oils against Culex quinquefasciatus Say larvae. Parasitol. Res. 2015, 114, 3835-3853. [CrossRef]

50. Perumalsamy, H.; Kim, N.-J.; Ahn, Y.-J. Larvicidal activity of compounds isolated from Asarum heterotropoides against Culex pipiens pallens, Aedes aegypti, and Ochlerotatus togoi (Diptera: Culicidae). J. Med. Entomol. 2009, 46, 1420-1423. [CrossRef]

51. Ribeiro, W.H.F.; Vasconcelos, J.N.; Arriaga, A.M.C.; de Oliveira, M.C.F.; Andrade-Neto, M.; Lemos, T.L.G.; Santiago, G.M.P.; Nascimento, R.F.; Mafezoli, J. Tephrosia toxicaria Pers essential oil: Chemical composition and larvicidal activity. Nat. Prod. Commun. 2006, 1, 391-393. [CrossRef]

52. Magalhães, L.A.M.I.; Lima Mda, P.; Marques, M.O.; Facanali, R.; Pinto, A.C.; Tadei, W.P. Chemical composition and larvicidal activity against Aedes aegypti larvae of essential oils from four Guarea species. Molecules 2010, 15, 5734-5741.

53. Santos, T.G.; Dognini, J.; Begnini, I.M.; Rebelo, R.A.; Verdi, M.; Dalmarco, E.M. Chemical characterization of essential oils from Drimys angustifolia Miers (Winteraceae) and antibacterial activity of their major compounds. J. Braz. Chem. Soc. 2013, 24, 164-170. [CrossRef]

54. Jirovetz, L.; Bail, S.; Buchbauer, G.; Denkova, Z.; Slavchev, A.; Stoyanova, A.; Schmidt, E.; Geissler, M. Antimicrobial testings, gas chromatographic analysis and olfactory evaluation of an essential oil of hop cones (Humulus lupulus L.) from Bavaria and some of its main compounds. Sci. Pharm. 2006, 74, 189-201. [CrossRef]

55. Rather, M.A.; Dar, B.A.; Dar, M.Y.; Wani, B.A.; Shah, W.A.; Bhat, B.A.; Ganai, B.A.; Bhat, K.A.; Anand, R.; Qurishi, M.A. Chemical composition, antioxidant and antibacterial activities of the leaf essential oil of Juglans regia L. and its constituents. Phytomedicine 2012, 19, 1185-1190. [CrossRef] [PubMed]

56. Schmidt, J.M.; Noletto, J.A.; Vogler, B.; Setzer, W.N. Abaco bush medicine: Chemical composition of the essential oils of four aromatic medicinal plants from Abaco Island, Bahamas. J. Herbs Spices Med. Plants 2006, 12, 43-65. [CrossRef]

57. Kubo, I.; Fujita, K.; Kubo, A.; Nihei, K.; Ogura, T. Antibacterial activity of coriander volatile compounds against Salmonella choleraesuis. J. Agric. Food Chem. 2004, 52, 3329-3332. [CrossRef]

58. Liu, K.; Chen, Q.; Liu, Y.; Zhou, X.; Wang, X. Isolation and biological activities of decanal, linalool, valencene, and octanal from sweet orange oil. J. Food Sci. 2012, 77, C1156-C1161. [CrossRef]

59. Morandim-Giannetti, A.A.; Pin, A.R.; Pietro, N.A.S.; de Oliveira, H.C.; Mendes-Giannini, M.J.S.; Alecio, A.C.; Kato, M.J.; de Oliveira, J.E.; Furlan, M. Composition and antifungal activity against Candida albicans, Candida parapsilosis, Candida krusei and Cryptococcus neoformans of essential oils from leaves of Piper and Peperomia species. J. Med. Plants Res. 2010, 4, 1810-1814.

60. Venturi, C.R.; Danielli, L.J.; Klein, F.; Apel, M.A.; Montanha, J.A.; Bordignon, S.A.L.; Roehe, P.M.; Fuentefria, A.M.; Henriques, A.T. Chemical analysis and in vitro antiviral and antifungal activities of essential oils from Glechon spathulata and Glechon marifolia. Pharm. Biol. 2015, 53, 682-688. [CrossRef]

61. Ho, C.-L.; Hsu, K.-P.; Tseng, Y.-H.; Wang, E.I.-C.; Liao, P.-C.; Chouc, J.-C.; Linc, C.-N.; Sua, Y.-C. Composition and antimicrobial activities of the leaf essential oil of Machilus kusanoi from Taiwan. Nat. Prod. Commun. 2011, 6, 267-270. [CrossRef] [PubMed] 
62. Dai, D.N.; Chung, N.T.; Huong, L.T.; Hung, N.H.; Chau, D.T.M.; Yen, N.T.; Setzer, W.N. Chemical compositions, mosquito larvicidal and antimicrobial activities of essential oils from five species of Cinnamomum growing wild in north central Vietnam. Molecules 2020, 25, 1303. [CrossRef] [PubMed]

63. Ebadollahi, A.; Ziaee, M.; Palla, F. Essential oils extracted from different species of the Lamiaceae plants family as prospective bioagents against several detrimental pests. Molecules 2020, 25, 1556. [CrossRef] 